Check for updates

Cite this: RSC Adv., 2018, 8, 15448

Received 24th February 2018

Accepted 18th April 2018

DOI: $10.1039 / \mathrm{c} 8 \mathrm{ra} 01637 \mathrm{~b}$

rsc.li/rsc-advances

\section{One-pot sequential multicomponent reaction between in situ generated aldimines and succinaldehyde: facile synthesis of substituted pyrrole-3-carbaldehydes and applications towards medicinally important fused heterocycles $\uparrow$}

\begin{abstract}
Anoop Singh,,$_{+}^{a}$ Nisar A. Mir,,$_{+}^{\text {ad }}$ Sachin Choudhary, ${ }^{\text {a }}$ Deepika Singh, ${ }^{b}$ Preetika Sharma, ${ }^{c}$ Rajni Kant ${ }^{\mathrm{C}}$ and Indresh Kumar (D) *a

An efficient sequential multi-component method for the synthesis of $\mathrm{N}$-arylpyrrole-3-carbaldehydes has been developed. This reaction involved a proline-catalyzed direct Mannich reaction-cyclization sequence between succinaldehyde and in situ generated Ar/HetAr/indolyl-imines, followed by IBXmediated oxidative aromatization in one-pot operation. The practical utility of this procedure is shown at gram-scale and the synthesis of diverse bioactive fused heterocyclic scaffolds such as pyrroloquinoline, pyrrolo-oxadiazole, dihydro pyrroloquinoline, and pyrrolo-phenanthridine.
\end{abstract}

\section{Introduction}

Medium sized nitrogen heterocycles are privileged scaffolds present in numerous natural and unnatural compounds. ${ }^{1}$ Among them, the pyrrole core is present in many important classes of natural products and its derivatives have been used as valuable intermediates for the synthesis of many drugs that exhibit interesting biological activities (Fig. 1). ${ }^{2}$ In addition, functionalized pyrroles have shown wide applications in agrochemicals and flavor components, dyes, and functionalized materials. ${ }^{3}$ Over the past few decades, a number of elegant methods to access functionalized pyrroles have been reported, which includes classical methods, ${ }^{4}$ cycloadditions, ${ }^{5}$ multicomponent, ${ }^{6}$ metal-catalyzed reactions, ${ }^{7}$ and several others. ${ }^{8}$ Despite the extensive efforts, the synthesis of C3-functionalized pyrroles is probably the most difficult task and required a special strategy. ${ }^{9}$ In particular, the regiospecific synthesis of pyrroles endowed with aldehyde group at C3-position is still very rare. ${ }^{10}$ Pyrrole-3-carbaldehydes have mainly been synthesized by the use of sterically bulky triisopropylsilyl (TIPS) as

${ }^{a}$ Department of Chemistry, Birla Institute of Technology and Science, Pilani 333 031, Rajasthan, India. E-mail: indresh.chemistry@gmail.com; indresh.kumar@pilani. bits-pilani.ac.in

${ }^{b}$ Instrumentation Division, IIIM-CSIR Lab, Jammu 180 001, India

${ }^{c} X$-ray Crystallography Laboratory, Post-Graduate Department of Physics \& Electronics, University of Jammu, Jammu 180 006, India

${ }^{d}$ Department of Chemistry, Govt. Degree College for Pulwama-192301, J\&K, India $\dagger$ Electronic supplementary information (ESI) available. CCDC 1400572 and 1471798. For ESI and crystallographic data in CIF or other electronic format see DOI: $10.1039 / \mathrm{c} 8 \mathrm{ra} 01637 \mathrm{~b}$

$\ddagger$ Both the authors contributed equally. protecting group on the nitrogen of pyrrole followed by Vilsmeier-Hacck formylation and deprotection as a multistep process (eqn (1), Scheme 1), ${ }^{10 a}$ along with other direct/indirect methods. ${ }^{10 b-e}$ However, these reported approaches have one or more drawbacks, such as the requirement of specially designed substrates, multistep process with low yields, and harsh reaction conditions. Furthermore, none of these methods could directly yield 1,2-disubstituted pyrrole-3-carbaldehydes, to the best of our knowledge. The development of synthetic protocol, which directly produces the required functionality at the desired position of heterocyclic ring systems, has become a major contribution to the pharmaceutical industry. Thus, the development of modular and simple pot-economic protocol to strategically access substituted pyrrole-3-carbaldehydes from easily available materials is still in high demand. Notably,

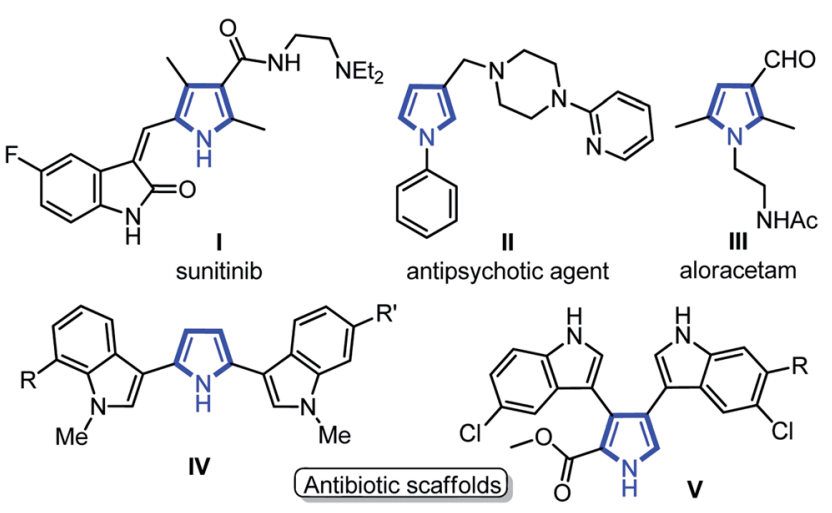

Fig. 1 Bioactive pyrroles and related derivatives. 
Earlier work: approach for selective C3-formylation ${ }^{\text {[ref. 10a] }}$

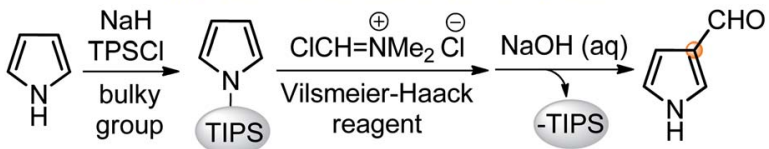

our previous work: two pot procedure $e^{[\text {ref. 13b] }}$

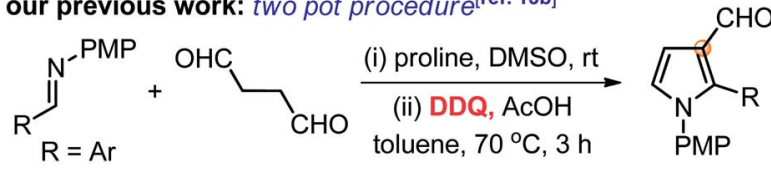

present work: one pot procedure

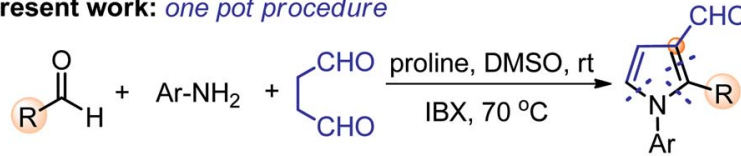

sequential multicomponent operation $R=A r$, HetAr, Indole

Scheme 1 Synthetic approaches for pyrrole-3-carbaldehydes.

aldehyde group at C3-position of pyrroles can readily participate in numerous name reactions, thus, holds significant promises to serve as a suitable intermediate to synthesize new medicinal agents and functionalized materials. ${ }^{11}$

In continuation to our efforts to utilize using linear dialdehydes, ${ }^{12}$ for the synthesis of medium-sized N-heterocycles under metal-free conditions, ${ }^{13}$ we recently developed a two-pot protocol for the direct synthesis of pyrrole-3-carbaldehyde

Table 1 Optimization of reaction conditions ${ }^{a}$

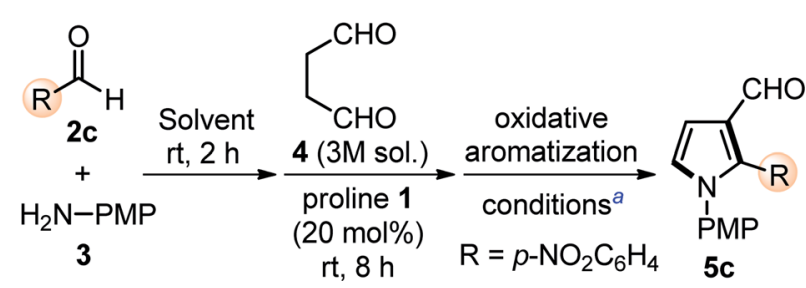

\begin{tabular}{|c|c|c|c|}
\hline Entry & Solvent & Conditions $^{a}$ & Yield $^{b}(\%$ \\
\hline 1 & DMSO & $\mathrm{K}_{2} \mathrm{~S}_{2} \mathrm{O}_{8}$ (1.2 equiv.), rt, $8 \mathrm{~h}$ & 35 \\
\hline 2 & DMSO & Oxone (1.2 equiv.), rt, $24 \mathrm{~h}$ & 40 \\
\hline 3 & DMF & Oxone (1.2 equiv.), rt, $24 \mathrm{~h}$ & 30 \\
\hline 4 & $\mathrm{CH}_{3} \mathrm{CN}$ & Oxone (1.2 equiv.), rt, $24 \mathrm{~h}$ & $<20$ \\
\hline 5 & DMSO & IBX (1.2 equiv.), rt, $6 \mathrm{~h}$ & 50 \\
\hline 6 & DMSO & IBX (1.2 equiv.), $50^{\circ} \mathrm{C}, 6 \mathrm{~h}$ & 64 \\
\hline 7 & DMSO & IBX (1.2 equiv.), $70^{\circ} \mathrm{C}, 4 \mathrm{~h}$ & 80 \\
\hline 8 & DMSO & IBX (1.2 equiv.), $90^{\circ} \mathrm{C}, 4 \mathrm{~h}$ & 75 \\
\hline $9^{c}$ & DMSO & IBX (1.2 equiv.), $70^{\circ} \mathrm{C}, 4 \mathrm{~h}$ & 58 \\
\hline 10 & DMF & IBX (1.2 equiv.), $70^{\circ} \mathrm{C}, 4 \mathrm{~h}$ & 43 \\
\hline $11^{d}$ & $\mathrm{CH}_{3} \mathrm{CN}$ & IBX (1.2 equiv.), $70^{\circ} \mathrm{C}, 4 \mathrm{~h}$ & 35 \\
\hline $12^{e}$ & DMSO & IBX (1.2 equiv.), $70^{\circ} \mathrm{C}, 4 \mathrm{~h}$ & 48 \\
\hline
\end{tabular}

${ }^{a}$ Unless otherwise indicated, the reaction was carried out with (i) aldehyde 2 (0.3 mmol), $p$-anisidine $3(0.3 \mathrm{mmol})$, succinaldehyde 4 (3 $\mathrm{M}$ aqueous sol., $0.9 \mathrm{mmol})$, proline $1(20 \mathrm{~mol} \%)$, solvent $(3.0 \mathrm{~mL})$; (ii) IBX (1.2 equiv.). ${ }^{b}$ Isolated yield of $5 \mathbf{c}$ refers to $2 \mathbf{c} .{ }^{c}$ Proline $\mathbf{1}$ (10 mol\%). ${ }^{d}$ EtOAc $(3.0 \mathrm{~mL})$ was added during IBX-mediated oxidative aromatization. ${ }^{e}$ Pyrrolidine (20 mol\%), $\mathrm{PhCO}_{2} \mathrm{H}(20 \mathrm{~mol} \%)$ were used in place of proline $\mathbf{1}$. from succinaldehyde and imines (eqn (2), Scheme 1). ${ }^{13 b}$ This method resulted in a quick synthesis of pyrrole-3-aldehydes, though, it required the pre-assembled imines and DDQ as a harsh and toxic reagent for oxidative aromatization. The clear synthetic potential and novelty of this method to suitably functionalized pyrroles prompted us to explore similar transformation in one-pot sequential multicomponent fashion under mild conditions. In addition, the social and environmental demands for more sustainable and practical synthetic protocols that need the use of less hazardous reagents/ conditions has also gained much attention of the scientific community. In this context, multicomponent reactions allow the rapid construction of novel libraries of pharmaceutically active compounds and marine alkaloids, thus, the development of such protocol is always applauded. ${ }^{14}$ Herein, we report a simple and most rational sequential multicomponent protocol for the synthesis of pyrrole-3-carbaldehydes via in situ imine formation between $\mathrm{Ar} / \mathrm{HetAr} /$ indole-aldehydes and $\mathrm{Ar}-$ $\mathrm{NH}_{2}$, followed by amine-catalyzed direct Mannich reactioncyclization with succinaldehyde, and IBX-mediated aromatization sequence in one-pot operation (eqn (3), Scheme 1). This improved method provides an easy access to pyrrole-3carbaldehydes under mild and non-toxic condition as compare to our previous two-pot protocol. In addition, in situ generated imines derived from indole-3-aldehydes have been explored for the first time for such transformations to yield indole-based medicinally important scaffolds.

\section{Results and discussion}

Based on our previous experience in this direction, we quickly optimized the designed reactions by choosing proline $\mathbf{1}$ (20 $\mathrm{mol} \%$ ) as a catalyst, $p$-nitrobenzaldehyde $\mathbf{2 c}$ as model substrates, along with $p$-anisidine 3, and succinaldehyde 4 (3 M aqueous sol.), in one-pot operation and the results are shown in Table 1. Initial experiments in DMSO as the choice of solvent along with the sequential addition of substrates, catalyst, and oxidant(s) gave $\mathbf{5 c}$ in low yield (entry 1 and 2, Table 1). Attempts were made to improve the yields by changing the solvents (entries 2-4, Table 1), however, failed. IBX as the oxidant, also soluble in DMSO, showed good efficiency for this one-pot protocol at rt (entry 5, Table 1) and at $50{ }^{\circ} \mathrm{C}$ (entry 6, Table 1). Gratifyingly, an additional improvement in yields $(80 \%)$ was observed when IBX-oxidation was carried out at $70{ }^{\circ} \mathrm{C}$ for $4 \mathrm{~h}$ (entry 7, Table 1). However, additional efforts to increase the reaction yield by further increment in reaction temperature (entry 8, Table 1), reduction in catalyst loading (entry 9, Table 1), varying the reaction medium (entries 10 and 11, Table 1), and changing the catalytic system (entry 12 , Table 1 ) were ineffective. Thus, we prefer to perform this one-pot sequential transformation with the optimized conditions (entry 7, Table 1).

The scope of the reaction was examined by employing various aromatic aldehydes and the results are summarized in Table 2. This one-pot sequential multicomponent protocol works well in case of aromatic aldehydes decorated with various groups (e.g. $-\mathrm{NO}_{2},-\mathrm{F},-\mathrm{Cl},-\mathrm{Br},-\mathrm{CN}$ and $\left.\mathrm{CF}_{3}\right)$ at the ortho-, meta-, or para-positions (entries 5a-5l, Table 2) and resulted in 2-aryl- 
Table 2 Substrate scope with respect to various Ar/HetAr-aldehydes $2^{a}$

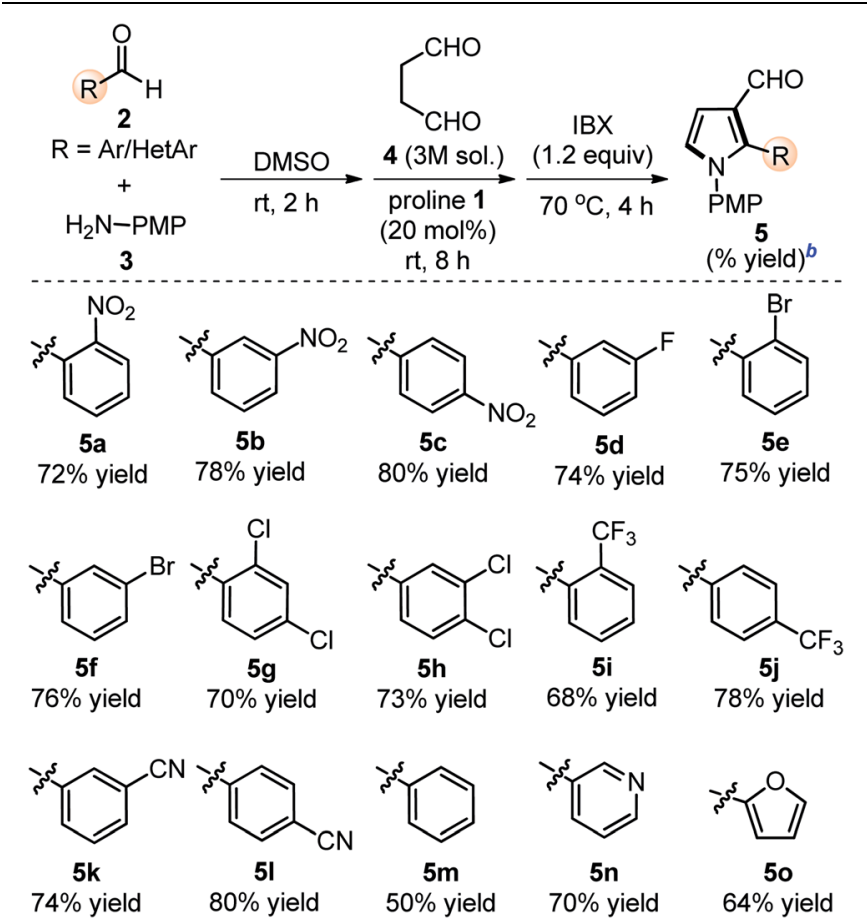

${ }^{a}$ Unless otherwise indicated, the reaction was carried out with (i) aldehyde $2(0.3 \mathrm{mmol}), p$-anisidine $3(0.3 \mathrm{mmol})$, DMSO $(3.0 \mathrm{~mL})$, rt, $2 \mathrm{~h}$, (ii) succinaldehyde 4 ( $3 \mathrm{M}$ aqueous sol., $0.9 \mathrm{mmol}$ ), proline 1 (20 mol\%), $8 \mathrm{~h}$, (iii) IBX (1.2 equiv.), $70{ }^{\circ} \mathrm{C}, 4 \mathrm{~h} .{ }^{b}$ Isolated yield of 5 refers to 2 ( $\leq 10 \%$ of aldehyde 2 was recovered in all the cases).

pyrrole-3-carbaldehydes in good to high yields (65-80\%). The reaction works well with in situ generated simple aryl imine (entry 5m, Table 2), as well as with hetero-aryl imines (entries 5n-5o, Table 2) with good yields. The feasibility of this protocol was also examined at gram scale of $2 \mathrm{c}(1.0 \mathrm{~g})$ with other reactants under standardized conditions and corresponding product 5c was obtained without much variation in yields $(1.60 \mathrm{~g}, 78 \%)$. The structure of $\mathbf{5 c}$ was further confirmed with single crystal X-ray diffraction analysis (Scheme 2). ${ }^{\mathbf{1 5}}$

\section{Indolyl-pyrroles synthesis}

The scope of this one-pot protocol was further was examined by employing various in situ generated imines derived from indole-

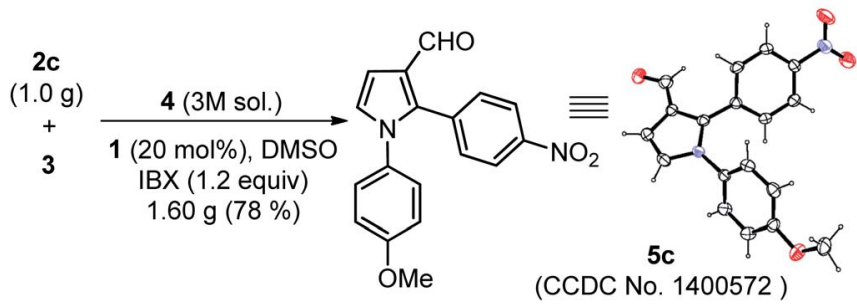

Scheme 2 Single-crystal X-ray analysis of 5c. Thermal ellipsoids are drawn at the $40 \%$ probability level.
Table 3 Substrate scope with respect to various indole-3-aldehydes $6^{a}$

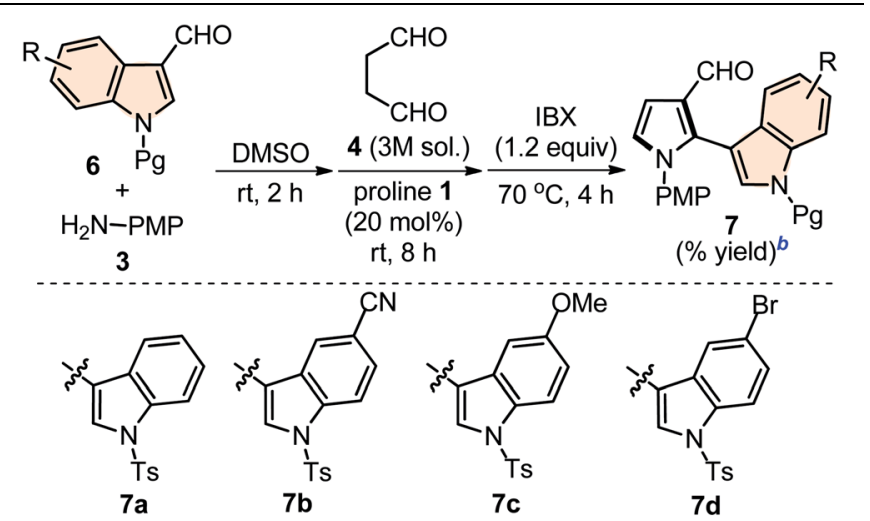<smiles>Cc1cn(C)c2ccc(Cl)cc12</smiles>

$73 \%$ yield

$70 \%$ yield

$70 \%$ yield
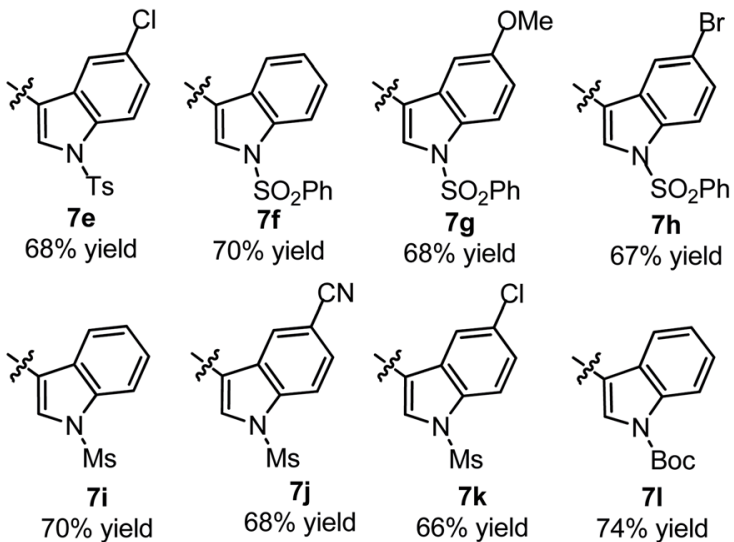

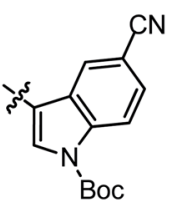

$7 \mathrm{~m}$

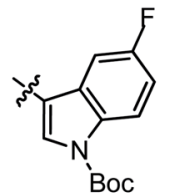

$7 n$ $67 \%$ yield<smiles>Cc1cn(C(=O)OC(C)(C)C)c2ccc(Br)cc12</smiles>

70<smiles>COc1ccc2c(c1)c(C)cn2C(=O)OCc1ccccc1</smiles>

$7 p$ $70 \%$ yield<smiles>Cn1cc(-c2c(C=O)ccn2-c2ccccc2O)c2ccccc21</smiles>

$7 q$

$60 \%$ yield<smiles>O=Cc1ccn(-c2ccc(Cl)cc2)c1-c1cn([125I])c2ccccc12</smiles><smiles>CC(C)(C)c1cn(Br)c2ccccc12</smiles>

$7 s$

n.r.

${ }^{a}$ Unless otherwise indicated, the reaction was carried out with (i) aldehyde 6 ( $0.3 \mathrm{mmol}), p$-anisidine $3(0.3 \mathrm{mmol})$, DMSO $(3.0 \mathrm{~mL})$, rt, $2 \mathrm{~h}$, (ii) succinaldehyde $4(3 \mathrm{M}$ aqueous sol., $0.9 \mathrm{mmol})$, proline 1 ( $20 \mathrm{~mol} \%$ ), $8 \mathrm{~h}$, (iii) IBX (1.2 equiv.), $70{ }^{\circ} \mathrm{C}, 4 \mathrm{~h} .{ }^{b}$ Isolated yield refers to $6(\leq 10 \%$ of aldehyde 6 was recovered in all the cases).

3-aldehydes 6. This extension to imines derived from indole-3aldehydes could be motivating as these units have not been utilized for similar direct Mannich reaction and can lead to new structural scaffolds. Indole and its derivatives are an important family of alkaloid compounds and most abundant heterocycles found in nature, which possess interesting biological 


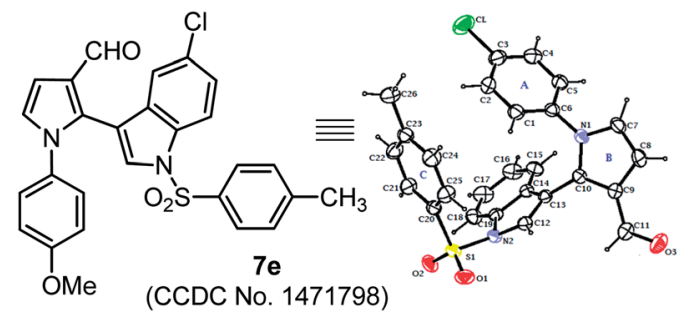

Fig. 2 Single-crystal X-ray analysis of 7e. Thermal ellipsoids are drawn at the $40 \%$ probability level.
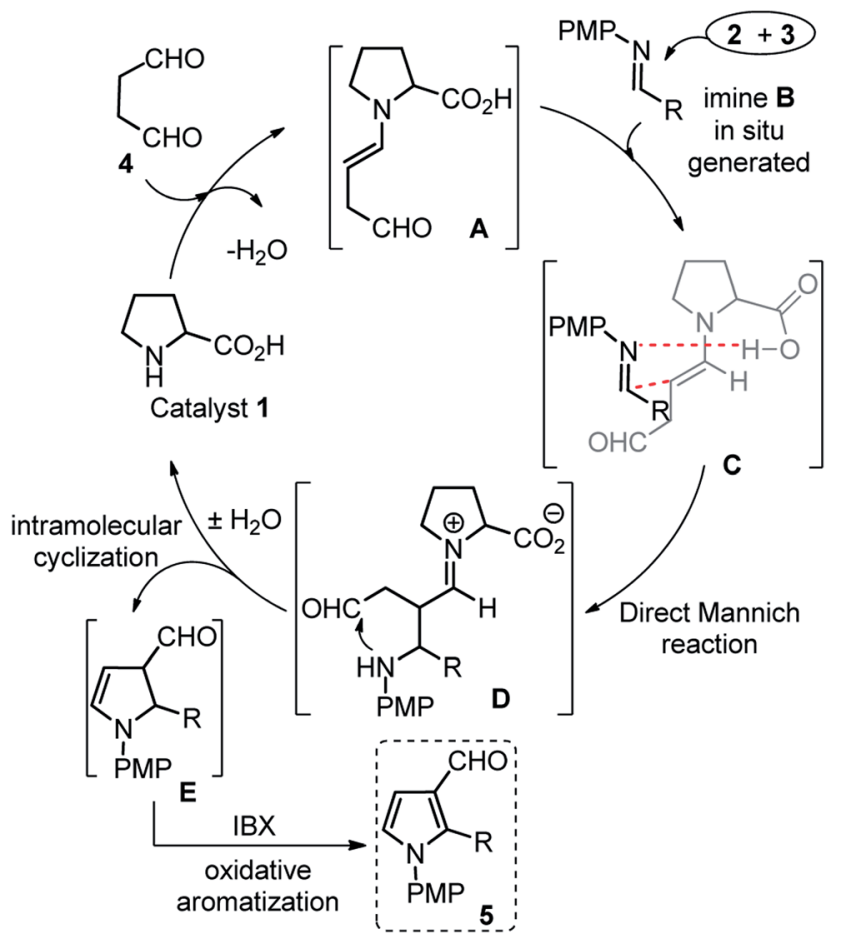

Scheme 3 Proposed reaction mechanism for this study.

activities. ${ }^{16}$ Moreover, indole-tethered pyrrole derivatives (for example IV and $\mathbf{V}$ in Fig. 1) found in several synthetic compounds and marine alkaloids that showed remarkable bioactivities, ${ }^{17}$ therefore, the synthesis of these compounds is quite interesting. In this context, a series of indolyl-pyrrole-3carbaldehydes $7 \mathbf{a}-7 \mathbf{p}$ were obtained with moderate to good yields, when $\mathrm{N}$-Ts, $\mathrm{Ms}, \mathrm{SO}_{2} \mathrm{Ph}$, Boc protected indole-3-aldehydes 6 were employed for this transformation with $p$-anisidine 3 and succinaldehyde $\mathbf{4}$ (entries $\mathbf{7 a - 7 p}$, Table 3 ). In addition, electron donating or withdrawing substitution on indole-ring did not alter the course of this transformation. Further, the reaction works quite well when other aryl-amines such as 2-amino-

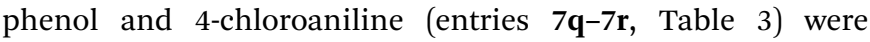
employed instead of $p$-anisidine 3 for this one-pot transformation with $N$-Ts-indol-3-aldehyde $\mathbf{6 a}$ and succinaldehyde 4 . However, reaction failed when the similar transformation was performed with $N$-benzyl-indole-3-aldehyde, probably because of low reactivity of imine (entry $7 \mathbf{s}$, Table 3 ). All compounds were well characterized by ${ }^{1} \mathrm{H}$ and ${ }^{13} \mathrm{C}$-NMR and mass-analysis. Single crystal X-ray diffraction analysis of $7 \mathrm{e}$ further established the product structure (Fig. 2). ${ }^{15}$ Based on our study, a stepwise mechanism is proposed to account for this reaction. As shown in Scheme 3, the enamine $\mathbf{A}$ in situ generated from succinaldehyde 4 and catalyst $\mathbf{1}$, reacts with in situ generated $N$ PMP-imine B via a direct Mannich reaction model $\mathbf{C}$ to produced Mannich product $\mathbf{D}$. The intermediate $\mathbf{D}$ underwent intramolecular cyclization to dihydropyrrole $\mathbf{E}$ with the simultaneous release of catalyst $\mathbf{1}$. In the same pot cyclic enamineintermediate $\mathbf{E}$ underwent IBX-mediated oxidative aromatization to afford pyrrole-3-carboxaldehyde 4.

\section{Synthetic applications}

Substituted pyrrole-3-aldehydes could participate as suitable intermediates for further functionalization to many important and complex scaffolds, therefore, we developed interesting and useful synthetic applications of these compounds. In this context, a rapid synthesis of pyrrolo[3,2-c]quinoline 8 was developed through reductive cyclization. This reaction proceeded via in situ amine formation through reduction of nitrogroup of $5 \mathrm{a} \mathrm{Fe} / \mathrm{NH}_{4} \mathrm{Cl}$ in EtOH : $\mathrm{H}_{2} \mathrm{O}(4: 1)$, which underwent intramolecular cyclization with aldehyde group in the same pot with good yields (eqn (1), Scheme 4). In another approach, the synthesis of pyrrole-dihydroquinoline $\mathbf{1 0}$ was accomplished through the reductive amination of $5 \mathbf{e}$ with $p$-anisidine 3 in presence of $\mathrm{NaBH}_{4}$ to generate in situ amine 9, which was

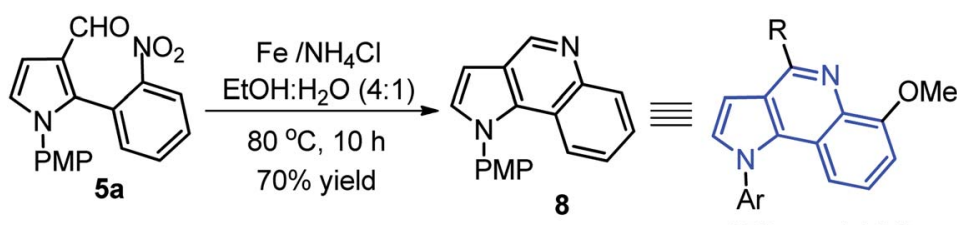

ATP-ase inhibitors<smiles>O=Cc1ccn(P(F)(F)(F)F)c1-c1ccccc1Br</smiles>

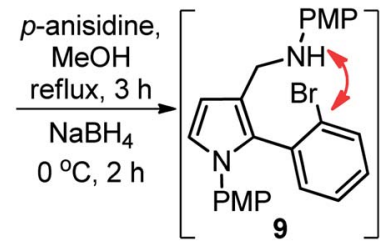

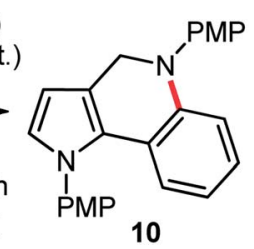

Scheme 4 Synthesis of pyrroloquinoline 8 and pyrrolo-dihydroquinoline 10 scaffolds. 

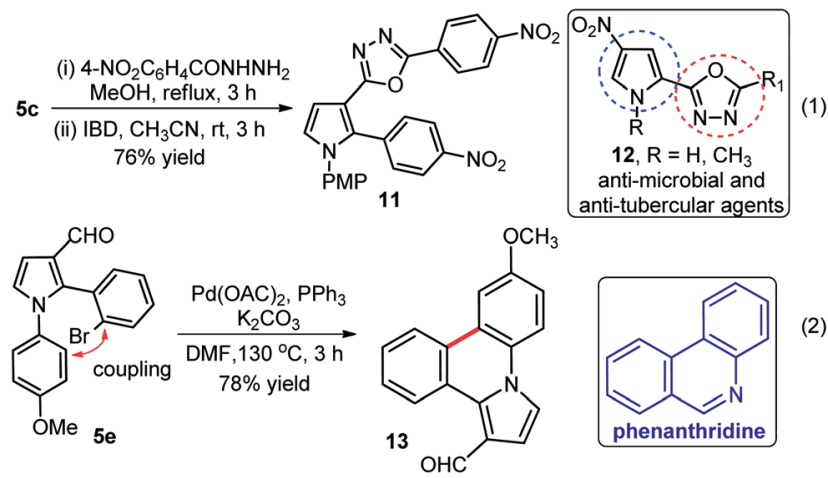

(2)

Scheme 5 Synthesis of pyrrole-oxadiazole 11, and pyrrole-phenanthridine 13 moieties.

further utilized for CuI-catalyzed intramolecular coupling (C-N) without purification to furnished 10 with high yield (87\%) over two steps (eqn (2), Scheme 4). ${ }^{18}$ The pyrroloquinoline moiety was found to be present in many natural/synthetic molecule with interesting bioactivity and our protocol may be better alternative to the previous procedure. ${ }^{19}$ The synthesized hybrid scaffolds resemble with various biologically active molecules such as pyrrolo[3,2-c]-quinoline derivative, an ATP-ase inhibitor, ${ }^{20 a}$ pyrrolo[2,3-c]-quinoline derivative, a natural product with acetylcholinesterase-inhibiting activity, ${ }^{20 b}$ and pyrrolo[3,4c] quinoline derivative, a potent 5-HT4R antagonist with analgesic action. ${ }^{20 c}$

Further applications of our method were shown; (i) synthesis of pyrrole-oxadiazole 11 in good yield (76\%) over two steps from compound $5 \mathbf{c}$ which was initially condensed with 4-nitrophenylhydrazide, followed by iodobenzene diacetate (IBD) mediated oxidative cyclization under the standardized conditions (eqn (1), Scheme 5), ${ }^{21}$ and (ii) rapid and high yielding (78\%) synthesis of pyrrole-phenanthridine $\mathbf{1 3}$ from 5e through intramolecular $\mathrm{C}-\mathrm{C}$ bond formation in presence $\mathrm{Pd}(\mathrm{OAc})_{2}, \mathrm{PPh}_{3}$ and $\mathrm{K}_{2} \mathrm{CO}_{3}$ in DMF at $130{ }^{\circ} \mathrm{C}$ as shown in (eqn (2), Scheme 5). Interestingly, 13 might exhibit interesting biological activities because phenanthridines serve as the core structure of natural products from Amaryllidaceae plants and received considerable attention from both chemists and biological scientists. ${ }^{22}$

\section{Conclusions}

In summary, we have developed a straight forwarded sequential multicomponent synthesis of substituted $\mathrm{N}$-aryl-pyrrole-3carbaldehydes. This one-pot protocol proceeds through proline-catalyzed Mannich reaction-cyclization sequence between succinaldehyde and imines, in situ generated from $\mathrm{Ar} /$ HetAr/indole-aldehydes with aromatic amines, followed by IBXmediated oxidative aromatization under mild conditions. Easy access to the starting materials and direct synthesis of pyrrole-3carbaldehydes under metal-free conditions renders this method potentially useful in organic synthesis. Synthetic applicability of the developed method was established through; (i) at gramscale synthesis, and (ii) the rapid access to the biologically important natural products analogous like-pyrrolo-quinoline, pyrrolo-oxadiazole, dihydro pyrroloquinoline, and pyrrolophenanthridine.

\section{Experimental}

\section{General remarks}

Unless otherwise stated, all reagents were purchased from commercial suppliers and used without further purification. All solvents employed in the reactions were distilled from appropriate drying agents prior to use. All reactions under standard conditions were monitored by thin-layer chromatography (TLC) on Merck silica gel $60 \mathrm{~F} 254$ pre-coated plates $(0.25 \mathrm{~mm})$. The column chromatography was performed on silica gel (100-200) using a mixture of hexane/EtOAc. Chemical yields refer to pure isolated substances. ${ }^{1} \mathrm{H}-\mathrm{NMR}$ spectra were recorded on a BRUKER-AV400 (400 MHz) spectrometer. Chemical shifts are reported in ppm from tetramethylsilane with the solvent resonance as the internal standard $\left(\mathrm{CDCl}_{3}=\delta 7.26\right.$ for ${ }^{1} \mathrm{H}$, and 77.0 for $\left.{ }^{13} \mathrm{C}-\mathrm{NMR}\right)$. Data are reported as follows: chemical shift, multiplicity ( $\mathrm{s}=$ singlet, $\mathrm{d}=$ doublet, $\mathrm{dd}=$ doublet of doublet, $\mathrm{t}$ $=$ triplet, $\mathrm{q}=$ quartet, $\mathrm{br}=$ broad, $\mathrm{m}=$ multiplet), coupling constants $(\mathrm{Hz})$ and integration. ${ }^{13} \mathrm{C}-\mathrm{NMR}$ spectra were recorded on a BRUKER-AV400 $(75 \mathrm{MHz})$ spectrometer with complete proton decoupling. HRMS were performed employing an ESI+ ionization method and TOF as an analyzer. Infrared (FT-IR) spectra were recorded on an ABB Bomen MB 3000 FTIR Spectrophotometer system using $\mathrm{KBr}$ pellets. Melting points were determined in open capillary tubes with an EZ-Melt automated melting point apparatus and may be incorrect.

\section{Typical procedure for the synthesis of pyrrole-3-carboxaldehydes}

To a stirred solution of Ar/HetAr-aldehyde $2(0.3 \mathrm{mmol})$ or $\mathrm{N}$ protected indole-3-aldehyde $6(0.3 \mathrm{mmol})$ in DMSO $(3.0 \mathrm{~mL})$ was added $p$-anisidine $3(0.3 \mathrm{mmol})$ and stirred initially for $2 \mathrm{~h}$ at rt. To this in situ generated-imine solution was added succinaldehyde $4(0.3 \mathrm{~mL}, 0.9 \mathrm{mmol}, 3 \mathrm{M}$ solution) and proline 1 $(7.0 \mathrm{mg}, 0.06 \mathrm{mmol})$ at the same temperature. The combined reaction mixture was stirred further for $8 \mathrm{~h}$ at $\mathrm{rt}$. At that time, IBX (100 mg, $0.36 \mathrm{mmol}, 1.2$ equiv.) was added to the reaction mixture and heated at $70^{\circ} \mathrm{C}$ for additional $3 \mathrm{~h}$. The reaction was cooled to room temperature quenched with $\mathrm{NaHCO}_{3}$ solution $(10 \%$ solution, $5 \mathrm{~mL})$ and extracted with EtOAc $(3 \times 6 \mathrm{~mL})$. The combined organic extracts were washed with brine, dried over $\mathrm{Na}_{2} \mathrm{SO}_{4}$ anhydrous, and concentrated under reduced pressure. Purification through silica gel column chromatography by eluting the mixture of hexane/EtOAc, gave pyrrole-3carbaldehydes 5 or 7 with $50-80 \%$ yields. In almost all the cases, we also obtained about $<10 \%$ initial starting aldehyde due to cleavage of corresponding imine under these conditions.

1-(4-Methoxyphenyl)-2-(2-nitrophenyl)-1H-pyrrole-3-carbaldehyde (5a). Reddish pasty liquid (70 mg, $72 \%)$; ${ }^{1} \mathrm{H}$ NMR $\left(400 \mathrm{MHz}, \mathrm{CDCl}_{3}\right)$ $\delta 3.77(\mathrm{~s}, 3 \mathrm{H}), 6.77(\mathrm{~d}, J=8.9 \mathrm{~Hz}, 2 \mathrm{H}), 6.87(\mathrm{~d}, J=3.0 \mathrm{~Hz}, 1 \mathrm{H})$, $6.94(\mathrm{~d}, J=3.0 \mathrm{~Hz}, 1 \mathrm{H}), 7.02(\mathrm{~d}, J=8.9 \mathrm{~Hz}, 2 \mathrm{H}), 7.45(\mathrm{~d}, J=$ $6.5 \mathrm{~Hz}, 1 \mathrm{H}), 7.54(\mathrm{t}, J=7.8 \mathrm{~Hz}, 1 \mathrm{H}), 7.62(\mathrm{t}, J=6.6 \mathrm{~Hz}, 1 \mathrm{H}), 7.97$ $(\mathrm{d}, J=8.1 \mathrm{~Hz}, 1 \mathrm{H}), 9.60(\mathrm{~s}, 1 \mathrm{H}) ;{ }^{13} \mathrm{C} \mathrm{NMR}\left(75 \mathrm{MHz}, \mathrm{CDCl}_{3}\right) \delta 55.4$, 109.1, 114.3 (2C), 124.4, 124.8, 125.1, 125.2, 126.9 (2C), 130.1, 
130.8, 132.7, 134.1, 135.8, 149.3, 159.1, 185.5; IR (KBr)/cm ${ }^{-1}$, 2932, 1666, 1520, 1350, 1296, 1034; HRMS (ESI): calcd for $\mathrm{C}_{18} \mathrm{H}_{14} \mathrm{~N}_{2} \mathrm{O}_{4}\left(\mathrm{MH}^{+}\right)$323.1032; found 323.1033.

1-(4-Methoxyphenyl)-2-(3-nitrophenyl)-1H-pyrrole-3-carbaldehyde (5b). Semi-solid (75 mg, 78\%); ${ }^{1} \mathrm{H}$ NMR (400 MHz, $\left.\mathrm{CDCl}_{3}\right) \delta 3.73$ (s, $3 \mathrm{H}), 6.77(\mathrm{~d}, J=8.8 \mathrm{~Hz}, 2 \mathrm{H}), 6.82(\mathrm{~d}, J=3.0 \mathrm{~Hz}, 1 \mathrm{H}), 6.87(\mathrm{~d}, J=$ $3.0 \mathrm{~Hz}, 1 \mathrm{H}), 6.97(\mathrm{~d}, J=8.0 \mathrm{~Hz}, 2 \mathrm{H}), 7.41-7.49(\mathrm{~m}, 2 \mathrm{H}), 8.01(\mathrm{t}, J$ $=1.4 \mathrm{~Hz}, 1 \mathrm{H}), 8.11(\mathrm{~d}, J=8.0 \mathrm{~Hz}, 1 \mathrm{H}), 9.65(\mathrm{~s}, 1 \mathrm{H}) ;{ }^{13} \mathrm{C} \mathrm{NMR}(75$ $\left.\mathrm{MHz} \mathrm{CDCl}_{3}\right) \delta 55.5,108.8,114.6(2 \mathrm{C}), 123.2,124.9,125.5,125.8$, 127.3 (2C), 129.2, 130.8, 131.1, 136.6, 138.2 , 147.9, 159.3, 186.0; IR (KBr) $/ \mathrm{cm}^{-1} 2920,1746,1680,1244,1172$; HRMS (ESI): calcd for $\mathrm{C}_{18} \mathrm{H}_{14} \mathrm{~N}_{2} \mathrm{O}_{4}\left(\mathrm{MH}^{+}\right)$323.1032; found 323.1033.

1-(4-Methoxyphenyl)-2-(4-nitrophenyl)-1 $\mathrm{H}$-pyrrole-3-carbaldehyde (5c). Pale yellow solid $\left(\mathrm{mp}=102-104{ }^{\circ} \mathrm{C}\right)(74 \mathrm{mg}, 80 \%) ;{ }^{1} \mathrm{H}$ NMR $(400$ $\left.\mathrm{MHz} \mathrm{CDCl}_{3}\right) \delta 3.82(\mathrm{~s}, 3 \mathrm{H}), 6.84(\mathrm{~d}, J=8.8 \mathrm{~Hz}, 2 \mathrm{H}), 6.89(\mathrm{~d}, J=$ $3.0 \mathrm{~Hz}, 1 \mathrm{H}), 6.94(\mathrm{~d}, J=3.0 \mathrm{~Hz}, 1 \mathrm{H}), 7.01(\mathrm{~d}, J=8.8 \mathrm{~Hz}, 2 \mathrm{H}), 7.35$ $(\mathrm{d}, J=8.8 \mathrm{~Hz}, 2 \mathrm{H}), 8.16(\mathrm{~d}, J=8.8 \mathrm{~Hz}, 2 \mathrm{H}), 9.73(\mathrm{~s}, 1 \mathrm{H}) ;{ }^{13} \mathrm{C} \mathrm{NMR}$ $\left(75 \mathrm{MHz}, \mathrm{CDCl}_{3}\right) \delta 55.4,109.0,114.6$ (2C), 123.4 (2C), 125.1, 126.1, 127.1 (2C), 130.9, 131.6 (2C), 135.9, 138.2, 147.4, 159.3, 186.0; IR (KBr) $/ \mathrm{cm}^{-1}$ 2933, 1724, 1660, 1249, 1174; HRMS (ESI): calcd for $\mathrm{C}_{18} \mathrm{H}_{14} \mathrm{~N}_{2} \mathrm{O}_{4}\left(\mathrm{MH}^{+}\right)$323.1032; found 323.1028.

2-(3-Fluorophenyl)-1-(4-methoxyphenyl)-1H-pyrrole-3carbaldehyde (5d). Yellow pasty liquid (65 mg, 74\%); ${ }^{1} \mathrm{H}$ NMR $\left(400 \mathrm{MHz}, \mathrm{CDCl}_{3}\right) \delta 3.80(\mathrm{~s}, 3 \mathrm{H}), 6.83(\mathrm{~d}, J=8.9 \mathrm{~Hz}, 2 \mathrm{H}), 6.86(\mathrm{~d}, J$ $=3.1 \mathrm{~Hz}, 1 \mathrm{H}), 6.89(\mathrm{~d}, J=2.8 \mathrm{~Hz}, 1 \mathrm{H}), 6.91(\mathrm{t}, J=1.8 \mathrm{~Hz}, 1 \mathrm{H})$, 7.00-7.06 (m, 4H), 7.28-7.32 (m, 1H), 9.70 (s, 1H); ${ }^{13} \mathrm{C}$ NMR (75 $\left.\mathrm{MHz}, \mathrm{CDCl}_{3}\right) \delta 55.4,107.8,114.3(2 \mathrm{C}), 115.7,117.9,124.6,125.2$, 126.8, 127.0, (2C), 129.8, 129.9, 131.3, 140.4, 158.9, 161.2, 186.6; IR (KBr) $/ \mathrm{cm}^{-1}, 2962,1720,1512,1247,1172$; HRMS (ESI): calcd for $\mathrm{C}_{18} \mathrm{H}_{14} \mathrm{FNO}_{2}\left(\mathrm{MH}^{+}\right)$296.1087; found 296.1070.

2-(2-Bromophenyl)-1-(4-methoxyphenyl)-1H-pyrrole-3carbaldehyde (5e). Red viscous liquid (80 mg, 75\%); ${ }^{1} \mathrm{H}$ NMR $\left(400 \mathrm{MHz}, \mathrm{CDCl}_{3}\right) \delta 3.77(\mathrm{~s}, 3 \mathrm{H}), 6.78(\mathrm{~d}, J=8.9 \mathrm{~Hz}, 2 \mathrm{H}), 6.86(\mathrm{~d}, J$ $=3.1 \mathrm{~Hz}, 1 \mathrm{H}), 6.92(\mathrm{~d}, J=3.0 \mathrm{~Hz}, 1 \mathrm{H}), 6.95(\mathrm{~d}, J=8.9 \mathrm{~Hz}, 2 \mathrm{H})$, 6.98-7.00 (m, 1H), 7.56 (dd, $J=7.9,4.3 \mathrm{~Hz}, 2 \mathrm{H}), 7.64(\mathrm{~d}, J=$ $8.3 \mathrm{~Hz}, 1 \mathrm{H}), 9.51(\mathrm{~s}, 1 \mathrm{H}) ;{ }^{13} \mathrm{C} \mathrm{NMR}\left(75 \mathrm{MHz}, \mathrm{CDCl}_{3}\right) \delta 55.4,107.9$, 114.2 (2C), 124.9, 125.1, 126.5 (2C), 126.9, 127.7, 129.7, 131.3, 134.0, 135.9, 136.1, 137.2, 158.9, 185.9; IR (KBr)/ $/ \mathrm{cm}^{-1}, 3016$, 1720, 1519, 1226, 1026; HRMS (ESI): calcd for $\mathrm{C}_{18} \mathrm{H}_{14} \mathrm{BrNO}_{2}$ $\left(\mathrm{MH}^{+}\right)$356.0286; found 356.0295.

2-(3-Bromophenyl)-1-(4-methoxyphenyl)-1H-pyrrole-3carbaldehyde (5f). Brown pasty liquid (81 $\mathrm{mg}, 76 \%) ;{ }^{1} \mathrm{H}$ NMR $\left(400 \mathrm{MHz}, \mathrm{CDCl}_{3}\right) \delta 3.81(\mathrm{~s}, 3 \mathrm{H}), 6.84(\mathrm{~d}, J=2.1 \mathrm{~Hz}, 1 \mathrm{H}), 6.86(\mathrm{~d}, J$ $=4.1 \mathrm{~Hz}, 2 \mathrm{H}), 6.89(\mathrm{~d}, J=3.0 \mathrm{~Hz}, 1 \mathrm{H}), 7.03(\mathrm{~d}, J=7.0 \mathrm{~Hz}, 2 \mathrm{H})$, $7.08-7.11(\mathrm{~m}, 1 \mathrm{H}), 7.17(\mathrm{t}, J=7.8 \mathrm{~Hz}, 1 \mathrm{H}) 7.41(\mathrm{t}, J=1.7 \mathrm{~Hz}, 1 \mathrm{H})$ 7.45-7.48 (m, 1H), $9.69(\mathrm{~s}, 1 \mathrm{H}) ;{ }^{13} \mathrm{C} \mathrm{NMR}\left(75 \mathrm{MHz}, \mathrm{CDCl}_{3}\right) \delta 55.5$, 108.0, 114.4 (2C), 122.2, 122.8, 124.7, 125.3, 127.1 (2C), 129.5, 129.7, 131.3, 131.6, 133.7, 137.2, 159.0, 186.5; IR $(\mathrm{KBr}) / \mathrm{cm}^{-1}$, 2985, 1728, 1519, 1373, 1242, 1049; HRMS (ESI): calcd for $\mathrm{C}_{18} \mathrm{H}_{14} \mathrm{BrNO}_{2}\left(\mathrm{MH}^{+}\right)$356.0286; found 356.0288.

2-(2,4-Dichlorophenyl)-1-(4-methoxyphenyl)-1H-pyrrole-3carbaldehyde (5g). Yellow oily liquid (77 mg, 70\%); ${ }^{1} \mathrm{H}$ NMR $\left(400 \mathrm{MHz}, \mathrm{CDCl}_{3}\right) \delta 3.78(\mathrm{~s}, 3 \mathrm{H}), 6.80(\mathrm{~d}, J=8.9 \mathrm{~Hz}, 2 \mathrm{H}), 6.86(\mathrm{~d}, J$ $=3.1 \mathrm{~Hz}, 1 \mathrm{H}), 6.94(\mathrm{~d}, J=2.9 \mathrm{~Hz}, 1 \mathrm{H}), 7.03(\mathrm{~d}, J=8.9 \mathrm{~Hz}, 2 \mathrm{H})$, $7.24(\mathrm{~d}, J=1.7 \mathrm{~Hz}, 2 \mathrm{H}), 7.40(\mathrm{~s}, 1 \mathrm{H}), 9.53(\mathrm{~s}, 1 \mathrm{H}) ;{ }^{13} \mathrm{C} \mathrm{NMR}(75$ $\left.\mathrm{MHz}, \mathrm{CDCl}_{3}\right) \delta 55.4,107.9,114.2(2 \mathrm{C}), 124.9,125.1,126.5$ (2C), $126.9,127.7,129.7,131.3,134.0,135.9,136.1,137.2$, 158.9,
185.9; IR (KBr)/cm ${ }^{-1}$, 2954, 1668, 1514, 1469, 1246, 1031; HRMS (ESI): calcd for $\mathrm{C}_{18} \mathrm{H}_{13} \mathrm{Cl}_{2} \mathrm{NO}_{2}\left(\mathrm{MH}^{+}\right)$346.0401; found 346.0408.

2-(3,4-Dichlorophenyl)-1-(4-methoxyphenyl)-1H-pyrrole-3carbaldehyde (5h). Yellow pasty liquid (80 mg, 73\%); ${ }^{1} \mathrm{H}$ NMR $\left(400 \mathrm{MHz}, \mathrm{CDCl}_{3}\right) \delta 3.70(\mathrm{~s}, 3 \mathrm{H}), 6.74(\mathrm{~d}, J=3.7 \mathrm{~Hz}, 2 \mathrm{H}), 6.75(\mathrm{~s}$, $1 \mathrm{H}), 6.78(\mathrm{~d}, J=3.1 \mathrm{~Hz}, 1 \mathrm{H}), 6.86-6.92(\mathrm{~m}, 3 \mathrm{H}), 7.14(\mathrm{~s}, 1 \mathrm{H}), 7.23$ $(\mathrm{t}, J=2.2 \mathrm{~Hz}, 2.0 \mathrm{~Hz}, 1 \mathrm{H}), 9.58(\mathrm{~s}, 1 \mathrm{H}) ;{ }^{13} \mathrm{C} \mathrm{NMR}\left(75 \mathrm{MHz}, \mathrm{CDCl}_{3}\right)$ $\delta 55.4,108.2,114.5$ (2C), 124.7, 125.5, 127.1 (2C), 129.3, 129.9, 130.2, 131.0, 132.5, 132.5, 133.0, 138.8, 159.1, 186.2; IR (KBr)/ $\mathrm{cm}^{-1}, 2962,1697,1514,1253,1031$; HRMS (ESI): calcd for $\mathrm{C}_{18} \mathrm{H}_{13} \mathrm{Cl}_{2} \mathrm{NO}_{2}\left(\mathrm{MH}^{+}\right)$346.0401; found 346.0406.

1-(4-Methoxyphenyl)-2-(2-(trifluoromethyl)phenyl)-1H-pyrrole3-carbaldehyde (5i). Yellow pasty liquid (75 mg, 68\%); ${ }^{1} \mathrm{H}$ NMR $\left(400 \mathrm{MHz}, \mathrm{CDCl}_{3}\right) \delta 3.75(\mathrm{~s}, 3 \mathrm{H}), 6.76(\mathrm{~d}, J=8.9 \mathrm{~Hz}, 2 \mathrm{H}), 6.85(\mathrm{~d}, J$ $=3.1 \mathrm{~Hz}, 1 \mathrm{H}), 6.91(\mathrm{~d}, J=3.0 \mathrm{~Hz}, 1 \mathrm{H}), 7.03(\mathrm{~d}, J=8.8 \mathrm{~Hz}, 2 \mathrm{H})$, $7.37(\mathrm{t}, J=4.5 \mathrm{~Hz}, 1 \mathrm{H}), 7.51(\mathrm{t}, J=4.9 \mathrm{~Hz}, 2 \mathrm{H}), 7.70(\mathrm{t}, J=4.6 \mathrm{~Hz}$, $1 \mathrm{H}), 9.41(\mathrm{~s}, 1 \mathrm{H}) ;{ }^{13} \mathrm{C} \mathrm{NMR}\left(75 \mathrm{MHz}, \mathrm{CDCl}_{3}\right) \delta 55.3,107.5,114.1$ (2C), 114.6, 117.6, 120.1, 121.5, 122.0, 124.8, 126.5, 127.0 (2C), 129.5, 131.1, 131.4, 134.0, 158.9, 186.0; IR (KBr)/. $\mathrm{cm}^{-1}, 2955$, 1666, 1520, 1311, 1250, 1119; HRMS (ESI): calcd for $\mathrm{C}_{19} \mathrm{H}_{14} \mathrm{~F}_{3} \mathrm{NO}_{2}\left(\mathrm{MH}^{+}\right)$346.1055; found 346.1057.

1-(4-Methoxyphenyl)-2-(4-(trifluoromethyl)phenyl)-1 H-pyrrole3-carbaldehyde (5j). Yellow pasty liquid (85 mg, 78\%); ${ }^{1} \mathrm{H}$ NMR $\left(400 \mathrm{MHz}, \mathrm{CDCl}_{3}\right) \delta 3.81(\mathrm{~s}, 3 \mathrm{H}), 6.84(\mathrm{~d}, J=9.0 \mathrm{~Hz}, 2 \mathrm{H}), 6.89(\mathrm{~d}, J$ $=3.1 \mathrm{~Hz}, 1 \mathrm{H}), 6.92(\mathrm{~d}, J=3.0 \mathrm{~Hz}, 1 \mathrm{H}), 7.02(\mathrm{~d}, J=8.9 \mathrm{~Hz}, 2 \mathrm{H})$, $7.33(\mathrm{~d}, J=8.1 \mathrm{~Hz}, 2 \mathrm{H}), 7.58(\mathrm{~d}, J=8.1 \mathrm{~Hz}, 2 \mathrm{H}), 9.70(\mathrm{~s}, 1 \mathrm{H}) ;{ }^{13} \mathrm{C}$ NMR (75 MHz, $\mathrm{CDCl}_{3}$ ) $\delta 55.4,108.3,114.5$ (2C), 124.9, 125.1, 125.2 (3C), 125.6, 127.1 (2C), 131.1 (2C), 131.2 (2C), 133.0, 159.1, 186.4; IR (KBr) $/ \mathrm{cm}^{-1}$, 2970, 1666, 1512, 1319, 1234; HRMS (ESI): calcd for $\mathrm{C}_{19} \mathrm{H}_{14} \mathrm{~F}_{3} \mathrm{NO}_{2}\left(\mathrm{MH}^{+}\right) 346.1055$; found 346.1053.

3-(3-Formyl-1-(4-methoxyphenyl)-1 H-pyrrol-2-yl) benzonitrile (5k). Yellow pasty liquid (67 mg, 74\%); ${ }^{1} \mathrm{H}$ NMR $(400 \mathrm{MHz}$, $\left.\mathrm{CDCl}_{3}\right) \delta 3.81(\mathrm{~s}, 3 \mathrm{H}), 6.84(\mathrm{~d}, J=8.8 \mathrm{~Hz}, 2 \mathrm{H}), 6.88(\mathrm{~d}, J=3.1 \mathrm{~Hz}$, $1 \mathrm{H}), 6.92(\mathrm{~d}, J=3.0 \mathrm{~Hz}, 1 \mathrm{H}), 7.01(\mathrm{~d}, J=8.9 \mathrm{~Hz}, 2 \mathrm{H}), 7.42-7.49$ $(\mathrm{m}, 3 \mathrm{H}), 7.41(\mathrm{~d}, J=7.0 \mathrm{~Hz}, 1 \mathrm{H}) 9.69(\mathrm{~s}, 1 \mathrm{H}) ;{ }^{13} \mathrm{C} \mathrm{NMR}(75 \mathrm{MHz}$, $\left.\mathrm{CDCl}_{3}\right) \delta 55.4,108.6,112.6,114.6$ (2C), 118.0, 124.8, 125.7, 127.1 (2C), 129.1, 130.8, 130.9, 131.9, 134.0, 135.1, 138.4, 159.2, 186.0; IR (KBr) $/ \mathrm{cm}^{-1}, 2932,2230,1659,1512,1443,1250$; HRMS (ESI): calcd for $\mathrm{C}_{19} \mathrm{H}_{14} \mathrm{~N}_{2} \mathrm{O}_{2}\left(\mathrm{MH}^{+}\right)$303.1134; found 303.1134.

4-(3-Formyl-1-(4-methoxyphenyl)-1H-pyrrol-2-yl) benzonitrile (51). Pink pasty liquid (72 mg, 80\%); ${ }^{1} \mathrm{H} \mathrm{NMR}\left(400 \mathrm{MHz}, \mathrm{CDCl}_{3}\right.$ ) $\delta 3.81(\mathrm{~s}, 3 \mathrm{H}), 6.85(\mathrm{~d}, J=8.9 \mathrm{~Hz}, 2 \mathrm{H}), 6.89(\mathrm{~d}, J=3.0 \mathrm{~Hz}, 1 \mathrm{H})$, $6.94(\mathrm{~d}, J=2.5 \mathrm{~Hz}, 1 \mathrm{H}), 7.01$ (d, $J=8.8 \mathrm{~Hz}, 2 \mathrm{H}), 7.32$ (d, $J=$ $8.3 \mathrm{~Hz}, 2 \mathrm{H}), 7.60$ (d, $J=8.3 \mathrm{~Hz}, 2 \mathrm{H}), 9.72(\mathrm{~s}, 1 \mathrm{H}) ;{ }^{13} \mathrm{C} \mathrm{NMR}(75$ $\left.\mathrm{MHz}, \mathrm{CDCl}_{3}\right) \delta 55.5,108.9,114.6(2 \mathrm{C}), 118.2,124.9,125.0,125.9$, 127.1 (2C), 131.4 (2C), 131.9 (2C), 134.1, 138.8, 141.7, 159.3, 186.0; IR (KBr) $/ \mathrm{cm}^{-1}$, 2962, 2229, 1712, 1519, 1242; HRMS (ESI): calcd for $\mathrm{C}_{19} \mathrm{H}_{14} \mathrm{~N}_{2} \mathrm{O}_{2}\left(\mathrm{MH}^{+}\right)$303.1134; found 303.1134.

1-(4-Methoxyphenyl)-2-phenyl-1 $H$-pyrrole-3-carbaldehyde (5m). Yellow pasty liquid (42 mg, 50\%); ${ }^{1} \mathrm{H}$ NMR (400 $\mathrm{MHz}, \mathrm{CDCl}_{3}$ ) $\delta 3.77(\mathrm{~s}, 3 \mathrm{H}), 6.80(\mathrm{~d}, J=8.8 \mathrm{~Hz}, 2 \mathrm{H}), 6.85(\mathrm{~d}, J=3.2 \mathrm{~Hz}, 1 \mathrm{H})$, $6.87(\mathrm{~d}, J=3.2 \mathrm{~Hz}, 1 \mathrm{H}), 7.00(\mathrm{~d}, J=8.8 \mathrm{~Hz}, 2 \mathrm{H}), 7.18-7.20(\mathrm{~m}$, $2 \mathrm{H}), 7.28-7.32(\mathrm{~m}, 3 \mathrm{H}), 9.67(\mathrm{~s}, 1 \mathrm{H}) ;{ }^{13} \mathrm{C} \mathrm{NMR}\left(75 \mathrm{MHz}, \mathrm{CDCl}_{3}\right)$ $\delta 55.3,107.6,114.2$ (2C), 124.3, 124.9, 127.0 (2C), 128.1 (2C), 128.4, 129.1, 130.9 (2C), 131.6, 142.4, 158.7, 187.0; IR (KBr)/ $\mathrm{cm}^{-1}, 2912$, 1710, 1672, 1244, 1174; HRMS (ESI): calcd for $\mathrm{C}_{18} \mathrm{H}_{15} \mathrm{NO}_{2}\left(\mathrm{MH}^{+}\right)$278.1181; found 278.1189. 
1-(4-Methoxyphenyl)-2-(pyridin-3-yl)-1H-pyrrole-3-carbaldehyde (5n). Red oily liquid (58 mg, 70\%); ${ }^{1} \mathrm{H}$ NMR (400 $\mathrm{MHz}, \mathrm{CDCl}_{3}$ ) $\delta 3.81(\mathrm{~s}, 3 \mathrm{H}), 6.85(\mathrm{~d}, J=8.9 \mathrm{~Hz}, 2 \mathrm{H}), 6.91(\mathrm{~d}, J=3.0 \mathrm{~Hz}, 1 \mathrm{H})$, $6.96(\mathrm{~d}, J=2.9 \mathrm{~Hz}, 1 \mathrm{H}), 7.04(\mathrm{~d}, J=8.9 \mathrm{~Hz}, 2 \mathrm{H}), 7.28(\mathrm{t}, J=$ $3.7 \mathrm{~Hz}, 1 \mathrm{H}), 7.54$ (d, $J=7.8 \mathrm{~Hz}, 1 \mathrm{H}), 8.5$ (bs, 1H), 8.58 (d, $J=$ $4.7 \mathrm{~Hz}, 1 \mathrm{H}), 9.72(\mathrm{~s}, 1 \mathrm{H}) ;{ }^{13} \mathrm{C} \mathrm{NMR}\left(75 \mathrm{MHz}, \mathrm{CDCl}_{3}\right) \delta 55.4,108.4$, 114.5 (2C), 122.9, 125.1, 125.7, 125.7, 127.3 (2C), 131.0, 137.8, 137.9, 149.4, 151.0, 159.2, 186.0; IR (KBr) $/ \mathrm{cm}^{-1}$, 2954, 1666, 1512, 1242, 1033; HRMS (ESI): calcd for $\mathrm{C}_{17} \mathrm{H}_{14} \mathrm{~N}_{2} \mathrm{O}_{2}\left(\mathrm{MH}^{+}\right)$ 279.1133; found 279.1140 .

2-(Furan-2-yl)-1-(4-methoxyphenyl)-1H-pyrrole-3-carbaldehyde (5o). Red oily liquid (51 mg, 64\%); ${ }^{1} \mathrm{H}$ NMR (400 $\mathrm{MHz}, \mathrm{CDCl}_{3}$ ) $\delta 3.85(\mathrm{~s}, 3 \mathrm{H}), 6.05(\mathrm{~d}, J=3.3 \mathrm{~Hz}, 1 \mathrm{H}), 6.37$ (d, $J=3.2 \mathrm{~Hz}, 1 \mathrm{H})$, $6.93(\mathrm{~d}, J=8.8 \mathrm{~Hz}, 2 \mathrm{H}), 7.18(\mathrm{~d}, J=8.8 \mathrm{~Hz}, 3 \mathrm{H}), 7.99$ (d, $J=$ $7.7 \mathrm{~Hz}, 1 \mathrm{H}), 8.05$ (d, $J=7.9 \mathrm{~Hz}, 1 \mathrm{H}), 10.08(\mathrm{~s}, 1 \mathrm{H}) ;{ }^{13} \mathrm{C} \mathrm{NMR}(75$ $\left.\mathrm{MHz}, \mathrm{CDCl}_{3}\right) \delta 55.5,108.1,111.2,111.7,114.3(2 \mathrm{C}), 126.0,127.2$ (2C), 127.9, 131.8, 133.1, 141.7, 143.4, 159.4, 187.4; IR (KBr)/ $\mathrm{cm}^{-1}, 2970,1682,1582,1466,1265,1011$; HRMS (ESI): calcd for $\mathrm{C}_{16} \mathrm{H}_{13} \mathrm{NO}_{3}\left(\mathrm{MH}^{+}\right)$268.0974; found 268.0980.

1-(4-Methoxyphenyl)-2-(1-tosyl-1H-indol-3-yl)-1H-pyrrole-3carbaldehyde (7a). Yellow solid (107 mg, 76\%, mp = 119-121 $\left.{ }^{\circ} \mathrm{C}\right) ;{ }^{1} \mathrm{H}$ NMR $\left(400 \mathrm{MHz}, \mathrm{CDCl}_{3}\right) \delta 2.37$ (s, 3H), $3.78(\mathrm{~s}, 3 \mathrm{H}), 6.71$ $(\mathrm{d}, J=8.9 \mathrm{~Hz}, 2 \mathrm{H}), 6.9(\mathrm{~d}, J=3.1 \mathrm{~Hz}, 1 \mathrm{H}), 6.97(\mathrm{~d}, J=2.4 \mathrm{~Hz}, 1 \mathrm{H})$, $7.02(\mathrm{~d}, J=8.9 \mathrm{~Hz}, 2 \mathrm{H}), 7.12-7.16(\mathrm{~m}, 1 \mathrm{H}), 7.23(\mathrm{~d}, J=8 \mathrm{~Hz}, 3 \mathrm{H})$, $7.28-7.32(\mathrm{~m}, 1 \mathrm{H}), 7.44$ (s, 1H), 7.63 (d, $J=8.3 \mathrm{~Hz}, 2 \mathrm{H}), 7.95$ (d, $=8.3 \mathrm{~Hz}, 1 \mathrm{H}), 9.58(\mathrm{~s}, 1 \mathrm{H}) ;{ }^{13} \mathrm{C} \mathrm{NMR}\left(75 \mathrm{MHz} \mathrm{CDCl}_{3}\right) \delta 29.6$, 55.4, 108.1, 111.5, 113.5, 114.3 (2C), 120.3, 123.9, 125.2, 125.7, 125.8, 126.5 (2C), 126.8 (2C), $127.1,129.9$ (2C), 130.2, 131.7, 133.3, 134.4, 134.7, 145.3, 158.9, 186.1; IR (KBr)/cm ${ }^{-1}, 2924$, 2854, 1659, 1597, 1512, 1173; HRMS (ESI): calcd for $\mathrm{C}_{27} \mathrm{H}_{22} \mathrm{~N}_{2} \mathrm{O}_{4} \mathrm{~S}\left(\mathrm{MH}^{+}\right)$471.1378; found 471.1382.

3-(3-Formyl-1-(4-methoxyphenyl)-1H-pyrrol-2-yl)-1-tosyl$\mathbf{1 H}$-indole-5-carbonitrile (7b). Brown solid (101 mg, 73\%, mp $\left.=123-125{ }^{\circ} \mathrm{C}\right) ;{ }^{1} \mathrm{H}$ NMR $\left(400 \mathrm{MHz}, \mathrm{CDCl}_{3}\right) \delta 2.40(\mathrm{~s}, 3 \mathrm{H}), 3.79(\mathrm{~s}$, $3 \mathrm{H}), 6.73(\mathrm{~d}, J=8.9 \mathrm{~Hz}, 2 \mathrm{H}), 6.90(\mathrm{~d}, J=3.1 \mathrm{~Hz}, 1 \mathrm{H}), 7.00-703$ $(\mathrm{m}, 3 \mathrm{H}), 7.28(\mathrm{~d}, J=8.0 \mathrm{~Hz}, 2 \mathrm{H}), 7.45(\mathrm{~d}, J=8.8 \mathrm{~Hz}, 1 \mathrm{H}), 7.52$ $(\mathrm{dd}, J=7.1 \mathrm{~Hz}, 1 \mathrm{H}), 7.64(\mathrm{~s}, 1 \mathrm{H}), 7.67$ (d, $J=8.4 \mathrm{~Hz}, 2 \mathrm{H}), 8.05$ (d, $J=8.1 \mathrm{~Hz}, 1 \mathrm{H}), 9.60(\mathrm{~s}, 1 \mathrm{H}) ;{ }^{13} \mathrm{C}-\mathrm{NMR}\left(75 \mathrm{MHz}, \mathrm{CDCl}_{3}\right) \delta 29.6$, 55.5, 109.1, 111.4, 114.4, 114.5 (2C), 125.4, 126.0, 126.5 (2C), 126.9 (2C), 128.1, 128.1, 129.1, 129.2, 129.8, 130.2, 130.3 (2C), 130.9, 131.3, 134.1, 136.0, 146.2, 159.1, 185.5; IR $(\mathrm{KBr}) / \mathrm{cm}^{-1}$, 2924, 2854, 2230, 1720, 1666, 1512, 1173; HRMS (ESI): calcd for $\mathrm{C}_{28} \mathrm{H}_{21} \mathrm{~N}_{3} \mathrm{O}_{4} \mathrm{~S}\left(\mathrm{MH}^{+}\right)$496.1331; found 496.1336.

2-(5-Methoxy-1-tosyl-1H-indol-3-yl)-1-(4-methoxyphenyl)-1Hpyrrole-3-carbaldehyde (7c). Red solid (105 mg, 70\%, mp = 127$\left.129{ }^{\circ} \mathrm{C}\right) ;{ }^{1} \mathrm{H}$ NMR $\left(400 \mathrm{MHz}, \mathrm{CDCl}_{3}\right) \delta 2.37(\mathrm{~s}, 3 \mathrm{H}), 3.64(\mathrm{~s}, 3 \mathrm{H})$, $3.78(\mathrm{~s}, 3 \mathrm{H}), 6.57(\mathrm{~d}, J=2.4 \mathrm{~Hz}, 1 \mathrm{H}), 6.71(\mathrm{~d}, J=8.9 \mathrm{~Hz}, 2 \mathrm{H})$, 6.88-6.9 (m, 2H), $6.98(\mathrm{~d}, J=2.7 \mathrm{~Hz}, 1 \mathrm{H}), 7.02(\mathrm{~d}, J=8.9 \mathrm{~Hz}, 2 \mathrm{H})$, $7.22(\mathrm{~d}, J=8.2 \mathrm{~Hz}, 2 \mathrm{H}), 7.43(\mathrm{~s}, 1 \mathrm{H}), 7.62(\mathrm{~d}, J=8.4 \mathrm{~Hz}, 2 \mathrm{H}), 7.82$ $(\mathrm{d}, J=9.1 \mathrm{~Hz}, 1 \mathrm{H}), 9.58(\mathrm{~s}, 1 \mathrm{H}) ;{ }^{13} \mathrm{C}-\mathrm{NMR}\left(75 \mathrm{MHz}, \mathrm{CDCl}_{3}\right)$ $\delta 21.5,55.4,55.5,102.0,108.2,111.5,114.3$ (2C), 114.5, 115.0, 125.6, 125.8, 126.3 (2C), 126.7 (2C), 127.9, 129.1, 129.9 (2C), 131.1, 131.8, 132.4, 134.7, 145.2, 156.8, 158.9, 186.1; IR (KBr)/ $\mathrm{cm}^{-1}, 2924,2854,1720,1659,1512,1173$; HRMS (ESI): calcd for $\mathrm{C}_{28} \mathrm{H}_{24} \mathrm{~N}_{2} \mathrm{O}_{5} \mathrm{~S}\left(\mathrm{MH}^{+}\right)$501.1484; found 501.1488.
2-(5-Bromo-1-tosyl-1H-indol-3-yl)-1-(4-methoxyphenyl)-1Hpyrrole-3-carbaldehyde (7d). Yellow solid (115 mg, 70\%, $\mathrm{mp}=$ 139-141 ${ }^{\circ} \mathrm{C}$ ); ${ }^{1} \mathrm{H}$ NMR (400 MHz, $\left.\mathrm{CDCl}_{3}\right) \delta 2.38(\mathrm{~s}, 3 \mathrm{H}), 3.79(\mathrm{~s}$, $3 \mathrm{H}), 6.72(\mathrm{~d}, J=8.9 \mathrm{~Hz}, 2 \mathrm{H}), 6.89(\mathrm{~d}, J=3.2 \mathrm{~Hz}, 1 \mathrm{H}), 6.97$ (d, $J=$ $2.5 \mathrm{~Hz}, 1 \mathrm{H}), 7.00$ (d, $J=8.9 \mathrm{~Hz}, 2 \mathrm{H}), 7.24$ (d, $J=8.0 \mathrm{~Hz}, 2 \mathrm{H}), 7.30$ $(\mathrm{d}, J=1.7 \mathrm{~Hz}, 2 \mathrm{H}), 7.37$ (d, $J=7.0 \mathrm{~Hz}, 1 \mathrm{H}), 7.45$ (s, 1H), 7.60 (d, $J$ $=8.4 \mathrm{~Hz}, 2 \mathrm{H}), 7.81(\mathrm{~d}, J=8.8 \mathrm{~Hz}, 1 \mathrm{H}), 9.57(\mathrm{~s}, 1 \mathrm{H}) ;{ }^{13} \mathrm{C} \mathrm{NMR}(400$ $\left.\mathrm{MHz}, \mathrm{CDCl}_{3}\right) \delta 29.6,55.5,108.5,110.9,114.4(2 \mathrm{C}), 115.0,117.5$, 123.1, 125.8, 125.9, 126.6 (2C), 126.8 (2C), 128.3, 128.3, 130.1 (2C), 131.5, 131.8, 132.3, 133.1, 134.4, 145.7, 159.1, 185.8; IR $(\mathrm{KBr}) / \mathrm{cm}^{-1}, 2924,2854,1720,1666,1512,1250,1119$; HRMS (ESI): calcd for $\mathrm{C}_{27} \mathrm{H}_{21} \mathrm{BrN}_{2} \mathrm{O}_{4} \mathrm{~S}\left(\mathrm{MH}^{+}\right)$549.0483; found 549.0488.

2-(5-Chloro-1-tosyl-1H-indol-3-yl)-1-(4-methoxyphenyl)-1Hpyrrole-3-carbaldehyde (7e). Brown solid (102 mg, 68\%, $\mathrm{mp}=$ 134-136 ${ }^{\circ} \mathrm{C}$ ); ${ }^{1} \mathrm{H}$ NMR (400 MHz, $\left.\mathrm{CDCl}_{3}\right) \delta 2.17(\mathrm{~s}, 3 \mathrm{H}), 3.58(\mathrm{~s}$, $3 \mathrm{H}), 6.52(\mathrm{~d}, J=8.9 \mathrm{~Hz}, 2 \mathrm{H}), 6.67(\mathrm{~d}, J=3.1 \mathrm{~Hz}, 1 \mathrm{H}), 6.76(\mathrm{dd}, J=$ $2.4 \mathrm{~Hz}, 1 \mathrm{H}), 6.80(\mathrm{~d}, J=8.9 \mathrm{~Hz}, 2 \mathrm{H}), 6.95(\mathrm{~d}, J=1.9 \mathrm{~Hz}, 1 \mathrm{H})$, 7.02-7.05 (m, 4H), 7.40 (d, $J=8.4 \mathrm{~Hz}, 2 \mathrm{H}), 7.65$ (d, $J=8.8 \mathrm{~Hz}$, $1 \mathrm{H}), 9.36(\mathrm{~s}, 1 \mathrm{H}) ;{ }^{13} \mathrm{C} \mathrm{NMR}\left(75 \mathrm{MHz}, \mathrm{CDCl}_{3}\right) \delta 29.7,55.5,108.4$, 111.0, 114.4 (2C), 114.6, 120.0, 125.6, 125.8, 125.9, 126.6 (2C), 126.8 (2C), 128.5, 130.0, 130.1 (2C), 131.4, 131.5, 132.5, 132.8, 134.5, 145.6, 159.1, 185.8; IR (KBr) $/ \mathrm{cm}^{-1}, 2924,2854,1666$, 1512, 1250, 1173; HRMS (ESI): calcd for $\mathrm{C}_{27} \mathrm{H}_{21} \mathrm{ClN}_{2} \mathrm{O}_{4} \mathrm{~S}\left(\mathrm{MH}^{+}\right)$ 505.0911; found 505.0916.

1-(4-Methoxyphenyl)-2-(1-(phenylsulfonyl)-1H-indol-3-yl)-1Hpyrrole-3-carbaldehyde (7f). Brown solid (96 mg, 70\%, $\mathrm{mp}=$ 117-119 ${ }^{\circ} \mathrm{C}$ ); ${ }^{1} \mathrm{H}$ NMR (400 MHz, $\mathrm{CDCl}_{3}$ ), $\delta 3.77$ (s, 3H), 6.69 (d, $J$ $=8.93 \mathrm{~Hz}, 2 \mathrm{H}), 6.89(\mathrm{~d}, J=3.1 \mathrm{~Hz}, 1 \mathrm{H}), 6.96(\mathrm{~d}, J=3.3 \mathrm{~Hz}, 1 \mathrm{H})$, $7.00(\mathrm{~d}, J=8.9 \mathrm{~Hz}, 2 \mathrm{H}), 7.12-7.16(\mathrm{~m}, 1 \mathrm{H}), 7.23(\mathrm{~d}, J=7.6 \mathrm{~Hz}$, 1H), 7.28-7.32 (m, 1H), 7.42-7.46 (m, 3H), 7.54-7.59 (m, 1H), $7.72-7.74(\mathrm{~m}, 2 \mathrm{H}), 7.96(\mathrm{~d}, J=8.3 \mathrm{~Hz}, 1 \mathrm{H}), 9.58(\mathrm{~s}, 1 \mathrm{H}) ;{ }^{13} \mathrm{C} \mathrm{NMR}$ (75 MHz, $\mathrm{CDCl}_{3}$ ), $\delta 55.4,108.1,111.7,113.4,114.3$ (2C), 120.3, 123.9, 125.3, 125.7, 126.5 (2C), 126.6 (2C), 127.1, 129.3 (2C), 130.2, 131.6, 132.2, 134.0, 134.4 (2C), 137.6, 158.8, 186.1; IR $(\mathrm{KBr}) / \mathrm{cm}^{-1}, 2932,2839,1720,1666,1512,1225,1180$; HRMS (ESI): calcd for $\mathrm{C}_{26} \mathrm{H}_{20} \mathrm{~N}_{2} \mathrm{O}_{4} \mathrm{~S}\left(\mathrm{MH}^{+}\right)$457.1222; found 457.1226.

2-(5-Methoxy-1-(phenylsulfonyl)-1H-indol-3-yl)-1-(4-methoxyphenyl)-1H-pyrrole-3-carbaldehyde (7g). Yellow viscous liquid (99 mg, 68\%); ${ }^{1} \mathrm{H}$ NMR (400 MHz, $\mathrm{CDCl}_{3}$ ) $\delta 3.64(\mathrm{~s}, 3 \mathrm{H}), 3.77$ (s, $3 \mathrm{H}), 6.56(\mathrm{~d}, J=2.4 \mathrm{~Hz}, 1 \mathrm{H}), 6.70(\mathrm{~d}, J=8.9 \mathrm{~Hz}, 2 \mathrm{H}), 6.88-6.91$ $(\mathrm{m}, 2 \mathrm{H}), 6.98(\mathrm{~d}, J=2.4 \mathrm{~Hz}, 1 \mathrm{H}), 7.00(\mathrm{~d}, J=8.9 \mathrm{~Hz}, 2 \mathrm{H}), 7.42-$ $7.45(\mathrm{~m}, 3 \mathrm{H}), 7.54-7.59(\mathrm{~m}, 1 \mathrm{H}), 7.71-7.73(\mathrm{~m}, 2 \mathrm{H}), 7.84(\mathrm{~d}, J=$ $9.1 \mathrm{~Hz}, 1 \mathrm{H}), 9.58(\mathrm{~s}, 1 \mathrm{H}) ;{ }^{13} \mathrm{C} \mathrm{NMR}\left(75 \mathrm{MHz}, \mathrm{CDCl}_{3}\right) \delta 55.4,55.5$, 102.0, 108.2, 111.8, 114.3 (2C), 114.5, 115.0, 125.6, 125.8, 126.3 (2C), 126.6 (2C), 127.8, 129.1, 129.3 (2C), 131.1, 131.7, 133.3, 134.0, 137.6, 156.9, 158.9, 186.1; IR (KBr) $/ \mathrm{cm}^{-1}, 2924,2854$, 1728, 1666, 1512, 1466, 1250, 1180; HRMS (ESI): calcd for $\mathrm{C}_{27} \mathrm{H}_{22} \mathrm{~N}_{2} \mathrm{O}_{5} \mathrm{~S}\left(\mathrm{MH}^{+}\right)$487.1327; found 487.1332.

2-(5-Bromo-1-(phenylsulfonyl)-1H-indol-3-yl)-1-(4-methoxyphenyl)1H-pyrrole-3-carbaldehyde (7h). Brownish yellow gummy liquid (107 mg, 67\%); ${ }^{1} \mathrm{H}$ NMR (400 MHz, $\mathrm{CDCl}_{3}$ ) $\delta 3.82$ (s, 3H), 3.76 (d, $J=8.9 \mathrm{~Hz}, 2 \mathrm{H}), 6.91(\mathrm{~d}, J=3.1 \mathrm{~Hz}, 1 \mathrm{H}), 7.00(\mathrm{~d}, J=3.1 \mathrm{~Hz}, 1 \mathrm{H})$, $7.3(\mathrm{~d}, J=8.9 \mathrm{~Hz}, 2 \mathrm{H}), 7.35(\mathrm{~d}, J=1.5 \mathrm{~Hz}, 1 \mathrm{H}), 7.42(\mathrm{~d}, J=6.9 \mathrm{~Hz}$, 1H), 7.48-7.51 (m, 3H), 7.60-7.65 (m, 1H), $7.74(\mathrm{~d}, J=7.3 \mathrm{~Hz}$, $2 \mathrm{H}), 7.86(\mathrm{~d}, J=8.8 \mathrm{~Hz}, 1 \mathrm{H}), 9.60(\mathrm{~s}, 1 \mathrm{H}) ;{ }^{13} \mathrm{C} \mathrm{NMR}(75 \mathrm{MHz}$, $\left.\mathrm{CDCl}_{3}\right) \delta 55.5,108.4,111.1,114.4(2 \mathrm{C}), 114.9,117.6,123.1,123.9$, 
125.8, 126.6 (2C), 126.7 (2C) 128.2, 128.4, 129.5 (2C), 131.4, 131.8, 132.1, 133.1, 134.3, 137.3, 159.0, 185.8; IR $(\mathrm{KBr}) / \mathrm{cm}^{-1}$, 2924, 2854, 1666, 1572, 1443, 1250, 1180; HRMS (ESI): calcd for $\mathrm{C}_{26} \mathrm{H}_{19} \mathrm{BrN}_{2} \mathrm{O}_{4} \mathrm{~S}\left(\mathrm{MH}^{+}\right)$535.0319; found 535.0325.

1-(4-Methoxyphenyl)-2-(1-(methylsulfonyl)-1H-indol-3-yl)1H-pyrrole-3-carbaldehyde (7i). Yellow pasty liquid (82 $\mathrm{mg}$, $70 \%) ;{ }^{1} \mathrm{H}$ NMR $\left(400 \mathrm{MHz}, \mathrm{CDCl}_{3}\right) \delta 3.07$ (s, 3H), $3.75(\mathrm{~s}, 3 \mathrm{H}), 6.77$ $(\mathrm{d}, J=9.0 \mathrm{~Hz}, 2 \mathrm{H}), 6.91(\mathrm{~d}, J=3.1 \mathrm{~Hz}, 1 \mathrm{H}), 7.01(\mathrm{~d}, J=2.4 \mathrm{~Hz}$, $1 \mathrm{H}), 7.08(\mathrm{~d}, J=9.0 \mathrm{~Hz}, 2 \mathrm{H}), 7.21-7.25(\mathrm{~m}, 1 \mathrm{H}), 7.31-7.34(\mathrm{~m}$, 2H), 7.35-7.39 (m, 1H), $7.90(\mathrm{~d}, J=8.4 \mathrm{~Hz}, 1 \mathrm{H}), 9.70(\mathrm{~s}, 1 \mathrm{H}) ;{ }^{13} \mathrm{C}$ NMR (75 MHz, $\mathrm{CDCl}_{3}$ ) $\delta 40.9,55.4,108.4,113.0,114.3$ (2C), 120.7, 124.2 , 125.3, 125.6, 125.7, 125.9, 126.5 (2C), 126.9, 130.2, 131.7, 132.9, 134.6, 159.1, 186.1; IR (KBr) $/ \mathrm{cm}^{-1} 2924,2854$, 1659, 1443, 1373, 1134; HRMS (ESI): calcd for $\mathrm{C}_{21} \mathrm{H}_{18} \mathrm{~N}_{2} \mathrm{O}_{4} \mathrm{~S}$ $\left(\mathrm{MH}^{+}\right)$395.1065; found 395.1070.

3-(3-Formyl-1-(4-methoxyphenyl)-1H-pyrrol-2-yl)-1-(methylsulfonyl)-1 $\boldsymbol{H}$-indole-5-carbonitrile (7j). Brownish solid $\left(85 \mathrm{mg}, 68 \%, \mathrm{mp}=114-116{ }^{\circ} \mathrm{C}\right) ;{ }^{1} \mathrm{H}$ NMR $\left(400 \mathrm{MHz}, \mathrm{CDCl}_{3}\right.$ ) $\delta 3.17(\mathrm{~s}, 3 \mathrm{H}), 3.77(\mathrm{~s}, 3 \mathrm{H}), 6.8(\mathrm{~d}, J=8.9 \mathrm{~Hz}, 2 \mathrm{H}), 6.93(\mathrm{~d}, J=$ $3.1 \mathrm{~Hz}, 1 \mathrm{H}), 7.03$ (d, J=2.6 Hz, 1H), 7.09 (d, $J=9.0 \mathrm{~Hz}, 2 \mathrm{H}), 7.53$ (m, 1H), 7.58 (d, J= 7.1 Hz, 1H), 7.99-8.03 (m, 2H), $9.73(\mathrm{~s}, 1 \mathrm{H})$; ${ }^{13} \mathrm{C}$ NMR $\left(75 \mathrm{MHz}, \mathrm{CDCl}_{3}\right.$ ) $\delta 41.8,55.5,107.9,109.6,111.5$, 114.1, 114.6 (2C), 118.6, 121.5, 125.8, 126.0, 126.5 (2C), 128.0, 128.4, 129.0, 131.3, 137.1, 141.7, 159.4, 185.6; IR $(\mathrm{KBr}) / \mathrm{cm}^{-1}$, 2924, 2854, 2230, 1659, 1512, 1381, 1180; HRMS (ESI): calcd for $\mathrm{C}_{22} \mathrm{H}_{17} \mathrm{~N}_{3} \mathrm{O}_{4} \mathrm{~S}\left(\mathrm{MH}^{+}\right)$419.0940; found 419.0946.

2-(5-Chloro-1-(methylsulfonyl)-1H-indol-3-yl)-1-(4-methoxyphenyl)-1H-pyrrole-3-carbaldehyde (7k). Yellowish solid $\left(85 \mathrm{mg}, 66 \%, \mathrm{mp}=126-128{ }^{\circ} \mathrm{C}\right) ;{ }^{1} \mathrm{H}$ NMR $\left(400 \mathrm{MHz}, \mathrm{CDCl}_{3}\right)$ $\delta 3.07(\mathrm{~s}, 3 \mathrm{H}), 3.77(\mathrm{~s}, 3 \mathrm{H}), 6.79(\mathrm{~d}, J=8.9 \mathrm{~Hz}, 2 \mathrm{H}), 6.91(\mathrm{~d}, J=$ $3.1 \mathrm{~Hz}, 1 \mathrm{H}), 7.01$ (d, $J=2.8 \mathrm{~Hz}, 1 \mathrm{H}), 7.08(\mathrm{~d}, J=8.9 \mathrm{~Hz}, 2 \mathrm{H}), 7.25$ $(\mathrm{d}, J=1.9 \mathrm{~Hz}, 1 \mathrm{H}), 7.31(\mathrm{~d}, J=6.8 \mathrm{~Hz}, 1 \mathrm{H}), 7.36(\mathrm{~s}, 1 \mathrm{H}), 7.82(\mathrm{~d}, J$ $=8.8 \mathrm{~Hz}, 1 \mathrm{H}), 9.70(\mathrm{~s}, 1 \mathrm{H}) ;{ }^{13} \mathrm{C} \mathrm{NMR}\left(75 \mathrm{MHz}, \mathrm{CDCl}_{3}\right) \delta 40.1$, 54.5, 107.8, 113.2, 113.4 (2C), 119.3, 121.6, 124.8, 124.9, 125.6 (2C), 126.0, 127.2, 129.2, 130.2, 130.4, 131.9, 135.6, 158.2, 184.8; IR (KBr)/cm ${ }^{-1}$, 2924, 2854, 1666, 1572, 1443, 1250, 1180; HRMS (ESI): calcd for $\mathrm{C}_{21} \mathrm{H}_{17} \mathrm{ClN}_{2} \mathrm{O}_{4} \mathrm{~S}\left(\mathrm{MH}^{+}\right)$429.0676; found 429.0682 .

tert-Butyl-3-(3-formyl-1-(4-methoxyphenyl)-1 $\mathrm{H}$-pyrrol-2-yl)-1 Hindole-1-carboxylate (7l). White solid, (92 mg, 74\%, $\mathrm{mp}=117-$ $\left.119{ }^{\circ} \mathrm{C}\right) ;{ }^{1} \mathrm{H}$ NMR $\left(400 \mathrm{MHz}, \mathrm{CdCl}_{3}\right), \delta 1.67(\mathrm{~s}, 9 \mathrm{H}), 3.74(\mathrm{~s}, 3 \mathrm{H})$, $6.66(\mathrm{~d}, J=9.0 \mathrm{~Hz}, 2 \mathrm{H}), 6.92(\mathrm{~d}, J=3.2 \mathrm{~Hz}, 1 \mathrm{H}), 6.98(\mathrm{~d}, J=$ $2.5 \mathrm{~Hz}, 1 \mathrm{H}), 7.07-7.16(\mathrm{~m}, 5 \mathrm{H}), 7.28-7.30(\mathrm{~m}, 1 \mathrm{H}), 7.57(\mathrm{~s}, 1 \mathrm{H})$, $8.12(\mathrm{~d}, J=8.1 \mathrm{~Hz}, 1 \mathrm{H}), 9.36(\mathrm{~s}, 1 \mathrm{H}) ;{ }^{13} \mathrm{C} \mathrm{NMR}\left(75 \mathrm{MHz}, \mathrm{CDCl}_{3}\right)$ $\delta 28.1$ (3C), 29.7, 55.44, 84.5, 108.0, 109.7, 114.3 (2C), 115.1, 120.1, 123.2, 124.9, 125.5, 125.9, 126.3 (2C), 127.2, 132.0, 134.7, 139.3, 149.2, 158.8, 186.7; IR (KBr) $/ \mathrm{cm}^{-1}, 2934,2860,1726$, 1666, 1512, 1250, 1157; HRMS (ESI): calcd for $\mathrm{C}_{25} \mathrm{H}_{24} \mathrm{~N}_{2} \mathrm{O}_{4}$ $\left(\mathrm{MH}^{+}\right)$417.1814; found 417.1820.

tert-Butyl-5-cyano-3-(3-formyl-1-(4-methoxyphenyl)-1H-pyrrol2-yl)-1H-indole-1-carboxylate (7m). Reddish brown solid (95 mg, $\left.72 \%, \mathrm{mp}=120-122{ }^{\circ} \mathrm{C}\right) ;{ }^{1} \mathrm{H}$ NMR $\left(400 \mathrm{MHz}, \mathrm{CDCl}_{3}\right) \delta 1.05(\mathrm{~s}$, $9 \mathrm{H}), 3.59(\mathrm{~s}, 3 \mathrm{H}), 6.53(\mathrm{~d}, J=8.9 \mathrm{~Hz}, 2 \mathrm{H}), 6.70(\mathrm{~d}, J=3.1 \mathrm{~Hz}, 1 \mathrm{H})$, $6.82(\mathrm{~d}, J=9.0 \mathrm{~Hz}, 3 \mathrm{H}), 7.07(\mathrm{~d}, J=3.7 \mathrm{~Hz}, 1 \mathrm{H}), 7.09(\mathrm{~s}, 1 \mathrm{H}), 7.32$ $(\mathrm{d}, J=1.4 \mathrm{~Hz}, 1 \mathrm{H}), 7.84(\mathrm{~d}, J=8.7 \mathrm{~Hz}, 1 \mathrm{H}), 9.40(\mathrm{~s}, 1 \mathrm{H}) ;{ }^{13} \mathrm{C} \mathrm{NMR}$ $\left(75 \mathrm{MHz}, \mathrm{CDCl}_{3}\right.$ ) $\delta 29.7$ (3C), 31.3, 55.5, 107.5, 109.1, 111.3, 114.5 (2C), 118.7, 125.5, 126.0, 126.5 (2C), 126.9, 128.1, 129.2, 129.9, 130.3, 131.0, 131.3, 134.1, 136.0, 146.2, 159.1, 185.6; IR (KBr)/ $\mathrm{cm}^{-1}, 2932,2862,2230,1666,1512,1173,1250$; HRMS (ESI): calcd for $\mathrm{C}_{26} \mathrm{H}_{23} \mathrm{~N}_{3} \mathrm{O}_{4}\left(\mathrm{MH}^{+}\right)$442.1767; found 442.1774.

tert-Butyl-5-fluoro-3-(3-formyl-1-(4-methoxyphenyl)-1 H-pyrrol-2-yl)-1H-indole-1-carboxylate (7n). Yellow solid (87 mg, 67\%, $\left.\mathrm{mp}=119-121{ }^{\circ} \mathrm{C}\right) ;{ }^{1} \mathrm{H}$ NMR $\left(400 \mathrm{MHz} \mathrm{CDCl}_{3}\right) \delta 1.66(\mathrm{~s}, 9 \mathrm{H})$, $3.75(\mathrm{~s}, 3 \mathrm{H}), 6.74-6.79(\mathrm{~m}, 3 \mathrm{H}), 6.90(\mathrm{~d}, J=3.1 \mathrm{~Hz}, 1 \mathrm{H}), 6.97-7.00$ $(\mathrm{m}, 2 \mathrm{H}), 7.13(\mathrm{~d}, J=8.9 \mathrm{~Hz}, 2 \mathrm{H}), 7.62(\mathrm{~s}, 1 \mathrm{H}), 8.07(\mathrm{~d}, J=5.9 \mathrm{~Hz}$, 1H), 9.68 (s, 1H), ${ }^{13} \mathrm{C}-\mathrm{NMR}\left(75 \mathrm{MHz}, \mathrm{CDCl}_{3}\right.$ ), $\delta 28.1$ (3C), 29.7, 55.4, 84.8, 105.6, 105.8, 108.2, 109.6, 112.8, 113.0, 114.4 (2C), 116.2, 125.6, 126.3 (2C), 128.6, 131.9, 134.0, 149.0, 158.9, 160.3, 186.5; IR (KBr)/cm ${ }^{-1}, 2924,2854,1736,1666,1450,1366,1250$, 1172; HRMS (ESI): calcd for $\mathrm{C}_{25} \mathrm{H}_{23} \mathrm{FN}_{2} \mathrm{O}_{4}\left(\mathrm{MH}^{+}\right)$435.1720; found 435.1726 .

tert-Butyl-5-bromo-3-(3-formyl-1-(4-methoxyphenyl)-1H-pyrrol-2-yl)-1H-indole-1-carboxylate (7o). White solid (82 $\mathrm{mg}, 68 \%$, $\left.\mathrm{mp}=129-131{ }^{\circ} \mathrm{C}\right) ;{ }^{1} \mathrm{H}$ NMR $\left(400 \mathrm{MHz}, \mathrm{CDCl}_{3}\right) \delta 1.66(\mathrm{~s}, 9 \mathrm{H})$, $3.02(\mathrm{~s}, 3 \mathrm{H}), 6.79(\mathrm{~d}, J=8.98 \mathrm{~Hz}, 2 \mathrm{H}), 6.90(\mathrm{~d}, J=3.12 \mathrm{~Hz}, 1 \mathrm{H})$, $6.98(\mathrm{~d}, J=3.9 \mathrm{~Hz}, 1 \mathrm{H}), 7.12(\mathrm{~d}, J=8.9 \mathrm{~Hz}, 2 \mathrm{H}), 7.22(\mathrm{~d}, J=$ $1.7 \mathrm{~Hz}, 1 \mathrm{H}), 7.36$ (d, $J=6.9 \mathrm{~Hz}, 1 \mathrm{H}), 7.75$ (s, 1H), 7.99 (d, $J=$ $8.4 \mathrm{~Hz}, 1 \mathrm{H}), 9.68(\mathrm{~s}, 1 \mathrm{H}),{ }^{13} \mathrm{C}$ NMR $\left(75 \mathrm{MHz}, \mathrm{CDCl}_{3}\right), \delta 28.1(3 \mathrm{C})$, 29.7, 55.5, 85.8, 108.2, 109.1, 114.4 (2C), 116.6, 122.8, 125.6, 125.9, 126.4 (2C) 127.8, 128.2, 131.1, 131.8, 133.7, 133.8, 148.8, 159.0, 186.3; IR (KBr) $/ \mathrm{cm}^{-1}$, 2932, 2862, 1736, 1680, 1458, 1373, 1157; HRMS (ESI): calcd for $\mathrm{C}_{25} \mathrm{H}_{23} \mathrm{BrN}_{2} \mathrm{O}_{4}\left(\mathrm{MH}^{+}\right)$495.0919; found 405.0924 .

tert-Butyl-3-(3-formyl-1-(4-methoxyphenyl)-1H-pyrrol-2-yl)-5methoxy-1H-indole-1-carboxylate (7p). Dark brown solid (89 mg, $\left.70 \%, \mathrm{mp}=124-126{ }^{\circ} \mathrm{C}\right) ;\left(400 \mathrm{MHz}, \mathrm{CDCl}_{3}\right), \delta 1.66(\mathrm{~s}, 9 \mathrm{H}), 3.64$ $(\mathrm{s}, 3 \mathrm{H}), 3.75(\mathrm{~s}, 3 \mathrm{H}), 6.51(\mathrm{~d}, J=2.4 \mathrm{~Hz}, 1 \mathrm{H}), 6.78(\mathrm{~d}, J=8.9 \mathrm{~Hz}$, $2 \mathrm{H}), 6.87(\mathrm{~d}, J=6.6 \mathrm{~Hz}, 1 \mathrm{H}), 6.93(\mathrm{~d}, J=3.2 \mathrm{~Hz}, 1 \mathrm{H}), 7.00(\mathrm{~d}, J=$ $2.4 \mathrm{~Hz}, 1 \mathrm{H}), 7.15$ (d, $J=9 \mathrm{~Hz}, 2 \mathrm{H}), 7.57$ (s, 1H), 7.99 (d, $J=$ $8.7 \mathrm{~Hz}, 1 \mathrm{H}), 9.71(\mathrm{~s}, 1 \mathrm{H}) ;{ }^{13} \mathrm{C}-\mathrm{NMR}\left(100 \mathrm{MHz}, \mathrm{CDCl}_{3}\right) \delta 28.1(3 \mathrm{C})$, 29.6, 55.4, 55.5, 84.3, 102.0, 108.0, 109.5, 114.3 (2C), 115.9, 125.4, 125.8, 126.1 (2C) 127.7, 129.6, 130.2, 132.2, 134.8, 149.1, 156.1, 158.8, 186.6; IR (KBr)/cm ${ }^{-1}, 2932,2862,1736,1666,1512$, 1250, 1157; HRMS (ESI): calcd for $\mathrm{C}_{26} \mathrm{H}_{26} \mathrm{~N}_{3} \mathrm{O}_{5}\left(\mathrm{MH}^{+}\right)$425.1171; found 425.1176 .

1-(2-Hydroxyphenyl)-2-(1-tosyl-1H-indol-3-yl)-1H-pyrrole-3carbaldehyde (7q). Light yellow solid ( $82 \mathrm{mg}, 60 \%, \mathrm{mp}=118-$ $\left.120{ }^{\circ} \mathrm{C}\right) ;{ }^{1} \mathrm{H}$ NMR $\left(400 \mathrm{MHz}, \mathrm{CDCl}_{3}\right) \delta 2.42(\mathrm{~s}, 3 \mathrm{H}), 6.95(\mathrm{~d}, J=$ $3.2 \mathrm{~Hz}, 1 \mathrm{H}), 7.07-7.01(\mathrm{~m}, 3 \mathrm{H}), 7.22-7.15(\mathrm{~m}, 3 \mathrm{H}), 7.25$ (d, $J=$ $7.4 \mathrm{~Hz}, 1 \mathrm{H}), 7.30$ (d, $J=8.5 \mathrm{~Hz}, 2 \mathrm{H}), 7.36-7.38(\mathrm{~m}, 1 \mathrm{H}), 7.46(\mathrm{~s}$, $1 \mathrm{H}), 7.67(\mathrm{~d}, J=8.4 \mathrm{~Hz}, 2 \mathrm{H}), 8.02(\mathrm{~d}, J=8.4 \mathrm{~Hz}, 1 \mathrm{H}), 9.63(\mathrm{~s}, 1 \mathrm{H})$; ${ }^{13} \mathrm{C} \mathrm{NMR}\left(75 \mathrm{MHz}, \mathrm{CDCl}_{3}\right.$ ) $\delta 21.6,108.7,111.0,113.6,120.1$, 124.02, 125.2, 125.4, 126.3, 126.4 (2C), 126.7 (2C), 126.9, 127.0, 129.3 (2C), 130.0 (2C), 132.8, 133.5, 134.4, 134.6, 137.1, 145.5, 186.0; IR (KBr)/cm ${ }^{-1}, 3458,2924,2854,1659,1497,1443,1088$; HRMS (ESI): calcd for $\mathrm{C}_{26} \mathrm{H}_{20} \mathrm{~N}_{2} \mathrm{O}_{4} \mathrm{~S}\left(\mathrm{MH}^{+}\right)$457.1222; found 457.1227.

1-(4-Chlorophenyl)-2-(1-tosyl-1H-indol-3-yl)-1H-pyrrole-3carbaldehyde (7r). Light pinkish solid ( $91 \mathrm{mg}, 64 \%, \mathrm{mp}=132-$ $\left.134{ }^{\circ} \mathrm{C}\right){ }^{1} \mathrm{H}$ NMR $\left(400 \mathrm{MHz}, \mathrm{CDCl}_{3}\right) \delta 2.35(\mathrm{~s}, 3 \mathrm{H}), 6.70(\mathrm{~m}, 1 \mathrm{H})$, $6.78(\mathrm{~s}, 1 \mathrm{H}), 6.87(\mathrm{~m}, 2 \mathrm{H}), 6.97(\mathrm{~d}, J=7.9 \mathrm{~Hz}, 1 \mathrm{H}), 7.25-7.10(\mathrm{~m}$, $4 \mathrm{H}), 7.37-7.28(\mathrm{~m}, 2 \mathrm{H}), 7.40(\mathrm{~d}, J=7.9 \mathrm{~Hz}, 1 \mathrm{H}), 7.56-7.50(\mathrm{~m}$, $2 \mathrm{H}), 7.90(\mathrm{~d}, J=8.4 \mathrm{~Hz}, 1 \mathrm{H}), 9.43(\mathrm{~s}, 1 \mathrm{H}),{ }^{13} \mathrm{C}$ NMR $(75 \mathrm{MHz}$, $\left.\mathrm{CDCl}_{3}\right) \delta 21.6,108.4,110.7,113.4,117.3,120.0,120.4,123.9$, 125.2, 125.2, 125.6, 126.1, 126.7 (2C), 127.0, 128.2, 129.9 (2C), 
130.1, 130.3, 134.2, 134.6, 135.1, 145.1, 151.8, 186.0; IR (KBr)/ $\mathrm{cm}^{-1}, 2924,2854,1651,1504,1443,1173$; HRMS (ESI): calcd for $\mathrm{C}_{26} \mathrm{H}_{19} \mathrm{ClN}_{2} \mathrm{O}_{3} \mathrm{~S}\left(\mathrm{MH}^{+}\right)$475.0883; found 475.0888.

1-(4-Methoxyphenyl)-1H-pyrrolo[3,2-c] quinoline (8). To a stirred solution of $5 \mathrm{a}(50 \mathrm{mg}, 0.15 \mathrm{mmol})$ in $\mathrm{EtOH}: \mathrm{H}_{2} \mathrm{O}(5 \mathrm{~mL}$, $4:$ 1) was added Fe-powder (86.9 mg, $1.55 \mathrm{mmol}, 10.0$ equiv.) and $\mathrm{NH}_{4} \mathrm{Cl}$ (100 mg, $1.8 \mathrm{mmol}, 12.0$ equiv.) and combined mixture was heated at $80{ }^{\circ} \mathrm{C}$ for $10 \mathrm{~h}$. The reaction progress was monitored by TLC, cooled and concentrated under reduced pressure once completed. The crude residue was extracted between EtOAc/ $\mathrm{NaHCO}_{3}$ solutions. Organic layer dried over $\mathrm{Na}_{2} \mathrm{SO}_{4}$ and evaporated under reduced pressure followed by silica-gel chromatography purification by eluting the mixture of hexane/EtOAc, gave pure product $\mathbf{8}$ as pasty yellow liquid (28 mg, 67\% yield). ${ }^{1} \mathrm{H}$ NMR (400 MHz, $\left.\mathrm{CDCl}_{3}\right) \delta 3.95(\mathrm{~s}, 3 \mathrm{H})$, $6.87(\mathrm{~d}, J=3.1 \mathrm{~Hz}, 1 \mathrm{H}), 7.09(\mathrm{~d}, J=8.6 \mathrm{~Hz}, 2 \mathrm{H}), 7.21$ (d, $J=$ $3.1 \mathrm{~Hz}, 1 \mathrm{H}), 7.23(\mathrm{~m}, 1 \mathrm{H}), 7.38$ (d, $J=9.1 \mathrm{~Hz}, 1 \mathrm{H}), 7.43$ (d, $J=$ $8.8 \mathrm{~Hz}, 2 \mathrm{H}), 7.53(\mathrm{t}, J=7.0 \mathrm{~Hz}, 1 \mathrm{H}), 8.19(\mathrm{~d}, J=8.3 \mathrm{~Hz}, 1 \mathrm{H}), 9.21$ $(\mathrm{s}, 1 \mathrm{H}) ;{ }^{13} \mathrm{C} \mathrm{NMR}\left(75 \mathrm{MHz}, \mathrm{CDCl}_{3}\right) \delta 55.6,103.3,114.8(2 \mathrm{C})$, 118.2, 120.6, 121.6, 125.3, 126.4, 128.5 (2C), 129.9, 130.2, 133.2, 134.6, 144.2, 146.1, 159.9; IR (KBr) $/ \mathrm{cm}^{-1}, 2924,1713,1512$, 1366, 1250, 1034; HRMS (ESI): calcd for $\mathrm{C}_{18} \mathrm{H}_{14} \mathrm{~N}_{2} \mathrm{O}\left(\mathrm{MH}^{+}\right)$ 275.1185; found 275.1190.

1,5-Bis(4-methoxyphenyl)-4,5-dihydro-1 $\mathrm{H}$-pyrrolo[3,2-c] quinoline (10). A mixture of 5e $(0.1 \mathrm{~g}, 0.28 \mathrm{mmol}, 1.0$ equiv. $)$ and $p$ anisidine 3 ( $0.030 \mathrm{~g}, 0.28 \mathrm{mmol}, 1.0$ equiv.) in methanol ( $3 \mathrm{~mL})$ was refluxed for $2 \mathrm{~h}$ at $80{ }^{\circ} \mathrm{C}$ and followed by reductive amination in the presence of $\mathrm{NaBH}_{4}$ at $0{ }^{\circ} \mathrm{C}$ to obtain intermediate product. This crude intermediate 9 ( $0.1 \mathrm{~g}, 0.22 \mathrm{mmol}, 1.0$ equiv.) was taken in oven-dried round-bottom flask dissolved in DMF (2 $\mathrm{mL}$ ), followed by the addition of base $\mathrm{K}_{2} \mathrm{CO}_{3}(61 \mathrm{mg}, 0.44 \mathrm{mmol}$, 2.0 equiv.), CuI ( $9 \mathrm{mg}, 20 \mathrm{~mol} \%)$, L-proline as ligand $(10 \mathrm{mg}$, $40 \mathrm{~mol} \%$ ). The resulting solution was stirred at $110{ }^{\circ} \mathrm{C}$ for $3 \mathrm{~h}$ under an $\mathrm{N}_{2}$ atmosphere. On completion, the residue was cooled to ambient temperature and then diluted with water ( 5 $\mathrm{mL})$ and extracted with EtOAc $(2 \times 5 \mathrm{~mL})$. The combined organic layers were dried over anhydrous $\mathrm{Na}_{2} \mathrm{SO}_{4}$ and evaporated to dryness. The crude residue was purified by column chromatography by eluting the mixture of hexane/EtOAc, afford 10 as yellow pasty liquid (70 mg, 87\% yield). ${ }^{1} \mathrm{H}$ NMR $(400 \mathrm{MHz}$, $\left.\mathrm{CDCl}_{3}\right) \delta 7.67(\mathrm{dd}, J=7.8,1.7 \mathrm{~Hz}, 1 \mathrm{H}), 7.56(\mathrm{dd}, J=7.9,1.2 \mathrm{~Hz}$, $1 \mathrm{H}), 7.28-7.33(\mathrm{~m}, 1 \mathrm{H}), 7.23(\mathrm{~d}, J=9.0 \mathrm{~Hz}, 2 \mathrm{H}), 7.08-7.14(\mathrm{~m}$, $1 \mathrm{H}), 6.93(\mathrm{~d}, J=2.4 \mathrm{~Hz}, 1 \mathrm{H}), 6.90(\mathrm{~d}, J=9.0 \mathrm{~Hz}, 2 \mathrm{H}), 6.74(\mathrm{~d}, J=$ $1.8 \mathrm{~Hz}, 1 \mathrm{H}), 6.72(\mathrm{~d}, J=9.0 \mathrm{~Hz}, 2 \mathrm{H}), 6.47$ (d, $J=8.9 \mathrm{~Hz}, 2 \mathrm{H}), 6.25$ $(\mathrm{d}, J=1.8 \mathrm{~Hz}, 1 \mathrm{H}), 5.80(\mathrm{~s}, 1 \mathrm{H}), 3.81(\mathrm{~s}, 3 \mathrm{H}), 3.70(\mathrm{~s}, 3 \mathrm{H}) ;{ }^{13} \mathrm{C}$ NMR (75 MHz, $\mathrm{CDCl}_{3}$ ) $\delta 55.5,55.7,56.2,109.2,114.2$ (2C), 114.5 (2C), 114.7 (2C), 118.1, 119.9, 121.9 (2C), 123.5, 126.7, 127.8, 128.1, 128.4, 132.9, 134.1, 141.3, 142.2, 151.9, 157.6; HRMS (ESI): calcd for $\mathrm{C}_{25} \mathrm{H}_{22} \mathrm{~N}_{2} \mathrm{O}_{2}\left(\mathrm{MH}^{+}\right)$383.1759; found 383.1765.

2-(1-(4-Methoxyphenyl)-2-(4-nitrophenyl)-1H-pyrrol-3-yl)-5(4-nitrophenyl)-1,3,4-oxadiazole (11). A mixture of 5c $(0.08 \mathrm{~g}$, $0.24 \mathrm{mmol}, 1.0$ equiv.) and 4-nitrobenzohydrazide (0.044 g, $0.24 \mathrm{mmol}, 1.0$ equiv.) in methanol $(3.0 \mathrm{~mL})$ was stirred for 2 hours under $80{ }^{\circ} \mathrm{C}$ and then concentrated in vacuo. The crude material was taken in $\mathrm{CH}_{3} \mathrm{CN}(3.0 \mathrm{~mL})$ and IBD $(0.08 \mathrm{~g}$, $0.24 \mathrm{mmol}, 1.0$ equiv.) was added stirred at room temperature for one hour. The mixture was then concentrated in vacuo. To the residue was added $\mathrm{H}_{2} \mathrm{O}(10 \mathrm{~mL})$ and the resulting mixture was extracted with ethylacetate $(10 \mathrm{~mL} \times 3)$. The organic layer was dried over $\mathrm{Na}_{2} \mathrm{SO}_{4}$ and concentrated. The residue was purified by column chromatography on silica gel by eluting the mixture of hexane/EtOAc, afford product $\mathbf{1 1}$ as yellow solid (92 mg, 76\% yield). ${ }^{1} \mathrm{H} \mathrm{NMR}\left(400 \mathrm{MHz}, \mathrm{CDCl}_{3}\right)(\mathrm{mp}=112-115$ $\left.{ }^{\circ} \mathrm{C}\right) \delta 8.33(\mathrm{~d}, J=9.0 \mathrm{~Hz}, 2 \mathrm{H}), 8.17(\mathrm{~d}, J=8.9 \mathrm{~Hz}, 2 \mathrm{H}), 8.08(\mathrm{~d}, J=$ $9.0 \mathrm{~Hz}, 2 \mathrm{H}), 7.52(\mathrm{~d}, J=8.9 \mathrm{~Hz}, 2 \mathrm{H}), 7.07$ (d, $J=3.0 \mathrm{~Hz}, 1 \mathrm{H}), 7.04$ $(\mathrm{d}, J=9.0 \mathrm{~Hz}, 2 \mathrm{H}), 7.01(\mathrm{~d}, J=3.0 \mathrm{~Hz}, 1 \mathrm{H}), 6.84(\mathrm{~d}, J=9.0 \mathrm{~Hz}$, 2H), 3.81 (s, 3H); ${ }^{13} \mathrm{C} \mathrm{NMR}\left(75 \mathrm{MHz}, \mathrm{CDCl}_{3}\right) \delta 55.5,108.0,109.8$, 114.5 (2C), 123.0 (2C), 124.3 (2C), 125.9, 127.1 (2C), 127.3 (2C), 129.4, 131.3, 132.0 (2C), 132.4, 137.0, 147.3, 149.2, 159.2, 161.6, 162.7; HRMS (ESI): calcd for $\mathrm{C}_{25} \mathrm{H}_{17} \mathrm{~N}_{5} \mathrm{O}_{6}\left(\mathrm{MH}^{+}\right)$484.1258; found 484.1263.

7-Methoxypyrrolo [1,2-f] phenanthridine-1-carbaldehyde (13). A clean oven-dried $10 \mathrm{~mL}$ round-bottom flask was charged with 5 e $(70 \mathrm{mg}, 0.19 \mathrm{mmol}, 1.0$ equiv.) in DMF ( $2 \mathrm{~mL})$, $\mathrm{K}_{2} \mathrm{CO}_{3}$ (54 mg, $0.39 \mathrm{mmol}, 2.0$ equiv.), ligand $\mathrm{PPh}_{3}$ (10 mg, $20 \mathrm{~mol} \%)$, and $\mathrm{Pd}(\mathrm{OAc})_{2}(5 \mathrm{mg}, 10 \mathrm{~mol} \%)$. The resulting solution was stirred at $130{ }^{\circ} \mathrm{C}$ for $3 \mathrm{~h}$ under an $\mathrm{N}_{2}$ atmosphere. On completion, the reaction mass was cooled to ambient temperature and then diluted with water $(5 \mathrm{~mL})$ and extracted with EtOAc $(2 \times 5 \mathrm{~mL})$. The combined organic layers were dried over anhydrous $\mathrm{Na}_{2} \mathrm{SO}_{4}$ and evaporated to dryness. The crude residue so obtained was purified by column chromatography by eluting the mixture of hexane/EtOAc, afford $\mathbf{1 3}$ as white solid (43 mg, 78\% yield). ${ }^{1} \mathrm{H} \mathrm{NMR}\left(400 \mathrm{MHz}, \mathrm{CDCl}_{3}\right) \delta 10.26(\mathrm{~s}, 1 \mathrm{H})$, 9.49 (dd, $J=7.1,2.3 \mathrm{~Hz}, 1 \mathrm{H}), 8.33$ (dd, $J=7.3,2.2 \mathrm{~Hz}, 1 \mathrm{H}), 7.88$ $(\mathrm{d}, J=9.2 \mathrm{~Hz}, 1 \mathrm{H}), 7.85(\mathrm{~d}, J=2.7 \mathrm{~Hz}, 1 \mathrm{H}), 7.80(\mathrm{~d}, J=3.3 \mathrm{~Hz}$, $1 \mathrm{H}), 7.62-7.70(\mathrm{~m}, 2 \mathrm{H}), 7.24(\mathrm{~d}, J=3.3 \mathrm{~Hz}, 1 \mathrm{H}), 7.22$ (dd, $J=9.1$, $2.8 \mathrm{~Hz}, 1 \mathrm{H}), 3.99(\mathrm{~s}, 3 \mathrm{H}) ;{ }^{13} \mathrm{C} \mathrm{NMR}\left(75 \mathrm{MHz}, \mathrm{CDCl}_{3}\right) \delta 55.7,106.6$, $114.0,115.1$, 117.0, 117.2, 117.7, 119.0, 120.5, 122.2, 123.9, 125.3, 127.2, 127.4, 128.5, 128.7, 157.2, 185.1; HRMS (ESI): calcd for $\mathrm{C}_{18} \mathrm{H}_{13} \mathrm{BrNO}_{2}\left(\mathrm{MH}^{+}\right)$276.1024; found 276.1029.

\section{Conflicts of interest}

There are no conflicts to declare.

\section{Acknowledgements}

This work was supported by OPERA-grant of BITS Pilani and Department of Science \& Technology, Science and Engineering Board (DST-SERB) New Delhi (EMR/2016/005599). AS thanks to UGC New Delhi for Research Fellowships. Authors are also grateful for generous support from the DST-FIST support to Department of Chemistry at BITS-Pilani.

\section{Notes and references}

1 (a) J. A. Joule and K. Mills, Heterocyclic Chemistry, Blackwell, Oxford, 4th edn, 2000; (b) T. Eicher and S. Hauptmann, The Chemistry of Heterocycles, Wiley-VCH, Weinheim, 2003; (c) A. R. Katrizky and A. F. Pozharskii, Handbook of Heterocyclic Chemistry, Pergamon, Amsterdam, 2nd edn, 2000; (d) I. S. Young, P. D. Thornton and A. Thompson, Nat. Prod. Rep., 2010, 27, 1801; (e) M. Baumann, 
I. R. Baxendale, S. V. Ley and N. Nikbin, Beilstein J. Org. Chem., 2011, 7, 442.

2 (a) Pyrroles, The Chemistry of Heterocyclic Compounds part I., ed. R. A. Jones, Wiley, New York, 1990, vol. 48; (b) H. Fan, J. Peng, M. T. Hamann and J.-F. Hu, Chem. Rev., 2008, 108, 264; (c) V. Bhardwaj, D. Gumber, V. Abbot, S. Dhiman and P. Sharma, RSC Adv., 2015, 46, 15233.

3 (a) M. E. Mason, B. Johnson and M. Hamming, J. Agric. Food Chem., 1966, 14, 454; (b) M. M. M. Raposo, A. M. C. Fonseca, M. C. R. Castro, M. Belsley, M. F. S. Cardoso, L. M. Carvalho and P. J. Coelho, Dyes Pigm., 2011, 91, 62; (c) M. Takase, N. Yoshida, T. Narita, T. Fujio, T. Nishinaga and M. Iyoda, RSC Adv., 2012, 2, 3221; (d) M. M. Wienk, M. Turbiez, J. Gilot and R. A. J. Janssen, Adv. Mater., 2008, 20, 2556; (e) Y. Wang, G. A. Sotzing and R. A. Weiss, Chem. Mater., 2008, 20, 2574; (f) S. K. Ibrahim, X. M. Liu, C. Tard and C. J. Pickett, Chem. Commun., 2007, 1535; (g) S. J. Higgins, Chem. Soc. Rev., 1997, 26, 247; (h) C.-F. Lee, L.-M. Yang, T.-Y. Hwu, A.-S. Feng, J.-C. Tseng and T.-Y. Luh, J. Am. Chem. Soc., 2000, 122, 4992; (i) C. D'Silva and D. A. Walker, J. Org. Chem., 1998, 63, 6715; (j) A. Berlin, B. Vercelli and G. Zotti, Polym. Rev., 2008, 48, 493.

4 For recent reviews, see (a) V. Estevez, M. Villacampa and J. C. Menendez, Chem. Soc. Rev., 2010, 39, 4402; (b) J. Bergman and T. Janosik, Mod. Heterocycl. Chem., 2011, 1, 269; (c) X. Qi, X. Xu and C.-M. Park, Chem. Commun., 2012, 48, 3996.

5 A. Padwa and W. H. Pearson, Synthetic Applications of 1,3Dipolar Cycloaddition Chemistry Toward Heterocycles and Natural Products, Wiley, New York, 2002, vol. 59.

6 For reviews on multicomponent reactions, see (a) V. Estèvez, M. Villacampa and J. C. Menéndez, Chem. Soc. Rev., 2010, 39, 4402; (b) G. Balme, D. Bouyssi and N. Monteiro, Heterocycles, 2007, 73, 87.

7 For recent review see; A. V. Gulevich, A. S. Dudnik, N. Chernyak and V. Gevorgyan, Chem. Rev., 2013, 113, 3084. 8 (a) S. Chiba, Y. Wang, G. Lapointe and K. Narasaka, Org. Lett., 2008, 10, 313; (b) G. Minetto, L. F. Raveglia, A. Sega and M. Taddei, Eur. J. Org. Chem., 2005, 5277; (c) S. Thirumalairajan, B. M. Pearce and A. Thompson, Chem. Commun., 2010, 46, 1797; (d) S. Ngwerume and J. E. Camp, Chem. Commun., 2011, 47, 1857; (e) O. A. Attanasi, G. Favi, F. Mantellini, G. Moscatelli and S. Santeusanio, J. Org. Chem., 2011, 76, 2860; (f) M. Zhang and J. Zhang, Chem. Commun., 2012, 48, 6399; (g) L. Meng, K. Wu, C. Liu and A. Lei, Chem. Commun., 2013, 49, 5853; (h) V. Estevez, M. Villacampa and J. C. Menendez, Chem. Commun., 2013, 49, 591; (i) T. Wu, L. Pan, X. Xu and Q. Liu, Chem. Commun., 2014, 50, 1797; (j) Y. H. Xu, T. He, Q. C. Zhang and T. P. Loh, Chem. Commun., 2014, 50, 2784; (k) C. Zhou and D. Ma, Chem. Commun., 2014, 50, 3085; (l) H. P. Kalmode, K. S. Vadagaonkar, K. Murugan and A. C. Chaskar, New J. Chem., 2015, 39, 4631; (m) W. W. Tan and N. Yoshikai, Chem. Sci., 2015, 6, 6448; (n) X. Wu, K. Li, S. Wang, C. Liu and A. Lei, Org. Lett., 2016, 18, 56; (o) T. J. Cogswell, C. S. Donald and R. Marquez, Org. Biomol. Chem., 2016, 14, 183.
9 (a) C. P. Andrieux, P. Audebert, A. Merz and R. Schwarz, New J. Chem., 1990, 14, 637; (b) J. M. Méndez, B. Flores, F. León, M. E. Martinez, A. Vazquez, G. A. Garcia and M. Salmón, Tetrahedron Lett., 1996, 37, 4099; (c) N. P. Pavri and M. L. Trudell, J. Org. Chem., 1997, 62, 2649; (d) J. E. Beecher and D. A. Tirrell, Tetrahedron Lett., 1998, 39, 3927; (e) A. Bunrit, S. Sawadjoon, S. Tšupova, P. J. R. Sjöberg and J. S. M. Samec, J. Org. Chem., 2016, 81, 1450 .

10 (a) B. L. Bray, P. H. Mathies, R. Naef, D. R. Solas, T. T. Tidwell, D. R. Artis and J. M. Muchowski, J. Org. Chem., 1990, 55, 6317; For direct method (b) A. Hamdan and J. W. F. Wasley, Synth. Commun., 1983, 13, 741; For indirect methods (c) H. Dumoulin, F. Fabis, P. Dallemagne, S. Roult and M. Robba, Synth. Commun., 1994, 24, 1855; (d) P. Haldar, J. Guin and J. K. Ray, Tetrahedron Lett., 2005, 46, 1071; (e) A. R. Kelly, M. H. Kerrigan and P. J. Walsh, J. Am. Chem. Soc., 2008, 130, 4097.

11 (a) H. Jendralla, E. Baader, W. Bartmann, G. Beck, A. Bergmann, E. Granzer, B. Kerekjarto, K. Kesseler, R. Krause, W. Schubert and G. Wess, J. Med. Chem., 1990, 33, 61; (b) D. Dumoulin, S. Rault and M. Robba, J. Heterocycl. Chem., 1996, 33, 255; (c) R. He, L.-F. Zeng, Y. He, L. Wu, A. M. Gunawan and Z.-Y. Zhang, Chem. Commun., 2013, 49, 2064; (d) S. Valente, S. Tomassi, G. Tempera, S. Saccoccio, E. Agostinelli and A. Mai, J. Med. Chem., 2011, 54, 8228; (e) W. M. Sigmund, G. Weerasekera, C. Marestin, S. Styron, H. Zhou, M. Z. Elsabee, J. Rühe, G. Wegner and R. S. Duran, Langmuir, 1999, 15, 6423; $(f)$ F. Martinnelli, A. Palmieri and M. Petrini, Chem.-Eur. J., 2011, 17, 7183; (g) L. J. Perez, T. K. Karagounis, A. Hurley, B. L. Bassle and M. F. Semmelhack, Chem. Sci., 2014, 5, 151; (h) M. R. Rivero and S. L. Buchwald, Org. Lett., 2007, 9, 973; (i) M. S. Butler, J. Nat. Prod., 2004, 67, 2141; (j) M. Toganoh, Y. Kawabe and H. Furuta, J. Org. Chem., 2011, 76, 7618.

12 I. Kumar, P. Ramaraju, N. A. Mir and A. Singh, Org. Biomol. Chem., 2015, 13, 1280.

13 (a) I. Kumar, N. A. Mir, V. K. Gupta and Rajnikant, Chem. Commun., 2012, 48, 6975; (b) I. Kumar, N. A. Mir, P. Ramaraju and B. P. Wakhloo, $R S C A d v$., 2012, 2, 8922; (c) I. Kumar, P. Ramaraju, N. A. Mir, D. Singh, V. K. Gupta and Rajnikant, Chem. Commun., 2013, 49, 5645; (d) I. Kumar, N. A. Mir, P. Ramaraju, D. Singh, V. K. Gupta and Rajnikant, RSC Adv., 2014, 4, 34548; (e) P. Ramaraju, N. A. Mir, D. Singh, V. K. Gupta, R. Kant and I. Kumar, Org. Lett., 2015, 17, 5582; (f) N. A. Mir, S. Choudhary, P. Ramaraju, D. Singh and I. Kumar, $R S C A d v ., 2016,6$, 39741; (g) P. Ramaraju, N. A. Mir, D. Singh and I. Kumar, $R S C$ Adv., 2016, 6, 60422; (h) P. Ramaraju, N. A. Mir, D. Singh, P. Sharma, R. Kant and I. Kumar, Eur. J. Org. Chem., 2017, 3461.

14 (a) A. Shaabani, A. Maleki, H. Mofakham and H. R. Khavasi, J. Comb. Chem., 2008, 10, 883; (b) G. Guillena, D. J. Ramon and M. Yus, Tetrahedron: Asymmetry, 2007, 18, 693; (c) D. Tejedor, D. Gonzalez-Cruz, A. Santos-Exposito, 
J. J. Marrero-Tellado, P. de-Armas and F. Garcia-Tellado, Chem.-Eur. J., 2005, 11, 3502; (d) B. B. Toure and D. G. Hall, Chem. Rev., 2009, 109, 4439; (e) A. Dömling, W. Wang and K. Wang, Chem. Rev., 2012, 112, 3083; $(f)$ T. Guntreddi, B. K. Allam and K. N. Singh, Synlett, 2012, 2635; (g) S. R. Roy, P. S. Jadhavar, K. Seth, K. K. Sharma and A. K. Chakraborti, Synthesis, 2011, 2261; (h) H. Benjamin, S. Z. Rotstein, V. Rai and K. Y. Andrei, Chem. Rev., 2014, 114, 8323.

15 See ESI. $\dagger$

16 (a) R. J. Sundberg, The Chemistry of Indoles, Academic Press, New York, 1970; (b) G. W. Gribble, in Comprehensive Heterocyclic Chemistry II, ed. A. R. Katritzky, C. W. Ress, E. F. V. Scriven and C. W. Bird, Pergamon Press, Oxford, 1996, vol. 2, p. 207; (c) D. J. Faulkner, Nat. Prod. Rep., 2002, 19, 1; (d) G. Bartoli, G. Bencivenni and R. Dalpozzo, Chem. Soc. Rev., 2010, 39, 4449; (e) W. Gul and M. T. Hamann, Life Sci., 2005, 78, 442; (f) A. J. Kochanowska-Karamyan and M. T. Hamann, Chem. Rev., 2010, 110, 4489.

17 (a) K. A. McArthur, S. S. Mitchell, G. Tsueng, A. Rheingold, D. J. White, J. Grodberg, K. S. Lam and B. C. M. Potts, J. Nat. Prod., 2008, 71, 1732; (b) D. S. Black, M. C. Bowyer and N. Kumar, Tetrahedron, 1997, 53, 8573; (c) J. Lehuédé, B. Fauconneau, L. Barrier, M. Ourakow, A. Piriou and J. M. Vierfond, Eur. J. Med. Chem., 1999, 34, 991; (d) M. S. Mohamed, R. Kamel and S. S. Fatahala, Eur. J. Med. Chem., 2011, 46, 3022; (e) N. R. Williamson, P. C. Fineran, T. Gristwood, S. R. Chawrai, F. J. Leeper and G. P. C. Salmond, Future Microbiol., 2007, 2, 605; (f) J. Lavrado, R. Moreira and A. Paulo, Curr. Med. Chem., 2010, 17, 2348; $(g)$ A. Carbone, B. Parrino, P. Barraja, V. Spanò, G. Cirrincione, P. Diana, A. Maier, G. Kelter and H.-H. Fiebig, Mar. Drugs, 2013, 11, 643; (h) W. Zhang,
Z. Liu, S. Li, T. Yang, Q. Zhang, L. Ma, X. Tian, H. Zhang, C. Huang, S. Zhang, J. Ju, Y. Shen and C. Zhang, Org. Lett., 2012, 14, 3364.

18 (a) F. Zhou, J. Guo, J. Liu, K. Ding, S. Yu and Q. Cai, J. Am. Chem. Soc., 2012, 134, 14326; (b) Z. Wang, W. Bao and Y. Jiang, Chem. Commun., 2005, 2849.

19 R. L. Goff, A. M. Lawson, A. Daich and S. Comesse, Org. Biomol. Chem., 2013, 11, 1818.

20 (a) T. H. Brown, R. J. Ife, D. J. Keeling, S. M. Laing, C. A. Leach, M. E. Parsons, C. A. Price, D. R. Reavill and K. J. Wiggall, J. Med. Chem., 1990, 33, 527; (b) Y. Sangnoi, O. Sakulkeo, S. Yuenyongsawad, A. Kanjanaopas, K. Ingkaninan, A. Plubrukarn and K. Suwanborirux, Mar. Drugs, 2008, 6, 578; (c) G. Furlotti, M. A. Alisi, C. Apicella, A. C. de Joannon, N. Cazzolla, R. Costi, G. C. Crucitti, B. Garrone, A. Iacovo, G. Magarò, G. Mangano, G. Miele, R. Ombrato, L. Pescatori, L. Polenzani, F. Rosi, M. Vitiello and R. Di Santo, J. Med. Chem., 2012, 55, 9446.

21 (a) R. Y. Yang and L. X. Dai, J. Org. Chem., 1993, 58, 3301; (b) R. A. Rane, B. Pavankumar, S. D. Borhade and P. K. Khandare, Eur. J. Med. Chem., 2013, 70, 49; (c) A. Idhayadhulla, R. S. Kumar, A. J. A. Nasser and A. Manilal, Bull. Chem. Soc. Ethiop., 2012, 26, 429.

22 (a) S. Ghosal, K. S. Saini and A. W. Frahm, Phytochemistry, 1983, 22, 2305; (b) S. Ghosal, Y. Kumar, D. K. Chakrabarti, J. Lal and S. K. Singh, Phytochemistry, 1986, 25, 1097; (c) Z. Jin, Nat. Prod. Rep., 2009, 26, 363; (d) K. Merz, T. Muller, S. Vanderheiden, G. Eisenbrand, D. Marko and S. Brase, Synlett, 2006, 3461; (e) T. Ishikawa, Med. Res. Rev., 2001, 21, 61; $(f)$ H. Tsukamoto, S. Kondo, Y. Mukudai, T. Nagumo, A. Yasuda, Y. Kurihara, T. Kamatani and S. Shintani, Anticancer Res., 2011, 31, 2841. 